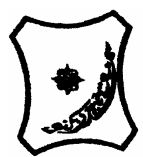

Bayero Journal of Pure and Applied Sciences, 10(1): 18 - 31

Received: October, 2016

Accepted: May, 2017

ISSN $2006-6996$

\title{
GEOMETRY OPTIMIZATION AND VIBRATIONAL FREQUENCIES OF TETRACENE MOLECULE IN GAS PHASE AND IN METHANOL BASED ON DENSITY FUNTIONAL THEORY AND RESTRICTED HARTREE-FOCK
}

\author{
${ }^{1}$ Gidado, A.S., ${ }^{2}$ Abubakar Maigari and ${ }^{1}$ Galadanci, G.S.M. \\ ${ }^{1}$ Department of Physics, Bayero University, Kano PMB 3011 \\ ${ }^{2}$ College of Education, Azare PMB 044, Bauchi State \\ *Correspondence author: asgidado.phy@buk.edu.ng
}

\begin{abstract}
ABSTRAC7
Tetracene is an organic semiconductor with chemical formula $\mathrm{C}_{18} \mathrm{H}_{12}$ used in organic field effecttransistor (OFET) and organic light emitting diode (OLED). In this work, the molecular geometry (optimized bond lengths and bond angles), vibrational frequencies and intensities, HOMO-LUMO Energy gap and Atomic charge distribution of the Tetracene molecule in gas phase and in solution were calculated and reported. Restricted Hartree-Fock (RHF) and Density Functional Theory (DFT) with different basis sets were employed for the task. Windows version of Gaussian 03 software was used to perform all the calculations. The results obtained show that the bond length obtained using RHF has the lowest average value of $1.072 \AA$ and that obtained using DFT has the lowest average value of $1.085 \AA$ in gas phase. In Methanol, it is observed that at RHF level, the lowest average value was 1.075A and at DFT level was 1.087A. This shows that the values are a bit higher in methanol than in gas phase which implies that the bonds will be slightly stronger in gas phase than in methanol. The strongest bonds in tetracene molecule are those of C20-H28, C23-H29 and C24H30 in both gas and methanol. The weakest bonds are those of C6-C12 and C6-C14.The bond angles were found to be so closed to $120^{\circ}$ at both levels of theory for all basis sets used suggesting that the molecule is planar benzene in which the C atoms are $s p^{2}$ hybridized. The calculated HOMOLUMO energy gap shows that the molecule will be slightly more stable in chemical reaction in gas phase than in methanol. DFT values of the energy gap appeared to be closer to the reported experimental value of $2.6 \mathrm{eV}$ than those obtained by RHF. The atomic charges distribution was found to be very sensitive to the basis sets which presumably occur due to polarization. From the results obtained for vibrational frequencies, it shows that tetracene molecule would be more stable in gas phase that in methanol as a result of no imaginary frequency found in gas phase. This confirms the stability of the molecule as stated in the results of HOMO-LUMO energy gap. The calculated vibrational frequencies show that the most intense frequency was obtained to be 924.9862 $\mathrm{cm}^{-1}$ at $146.7973 \mathrm{KM} / \mathrm{mole}$ by RHF/3-21G in gas phase while at B3L YP/3-21G, it has the most intense frequency of about $474.1260 \mathrm{~cm}^{-1}$ at $390.1077 .2845 \mathrm{MK} / \mathrm{mole}$ in methanol.
\end{abstract}

Keywords: Optimixation, Methanol, Gas, Frequency, Tetracene

\section{INTRODUCTION}

Organic semiconductor materials (OSM) such as oligoacenes, a subclass of polyaromatic hydrocarbon are made of fused of benzene rings joined in a linear arrangement ( Cappellin et. al., 2009) hold a great promising benefit as active elements in a variety of electronic and optoelectronic devices (Yumusak et. al., 2012) . In general, one of the most important advantages of organic materials instead of inorganic materials is its low cost, low temperature and solution-based processing. Among the organic compounds, polyacenes demonstrate a promising results as the ideal case due to their simple structure and they find several applications in organic thin films field effect transistors, organic light-emitting diode (OLEDs), solar cell, e.t.c . Also, acene ring based on tetracene molecule was reported to exhibit superconducting properties at low temperature. Moreover, Organic semiconductors have been intensely researched for two decades because they are key components in organic field-effect transistors (OFETs) which are necessary for next-generation electronics, such as flexible sensors. The term organic semiconductors implies that the materials are mostly made up by carbon and hydrogen atoms, with a few heteroatom such as sulfur, oxygen and nitrogen included and they show properties typically associated with a semiconductor materials (Anna and Heinz, 2015).

Conduction mechanisms for organic semiconductor are mainly through tunneling; hopping between localized states, mobility gaps, and phonon assisted hopping. Like inorganic semiconductors, organic semiconductors can be doped in order to change its conductivity. Although inorganic semiconductors such as silicon, germanium and gallium arsenide have been the backbone of semiconductor industry, for the past decade, demands for pervasive computing have led to a dramatic improvement in the performance of organic semiconductor. Organic semiconductors have been used as active elements in optoelectronic devices such as organic light emitting diodes (OLED), organic solar cells, and organic field effect transistors (OFET). There are many advantages of using organic semiconductors, such as easy fabrication, mechanical flexibility, and low cost. 
Organic semiconductors offer the ability to fabricate electronic device at lower temperature and over large areas on various flexible substrate such as plastic and paper. They can be processed using existing techniques used in semi conducting industry as well as in printing industries such as roll-to-roll manufacturing.

However, the mobility of organic semiconductor cannot match the performance of fieldeffect transistors based on single-crystalline inorganic semiconductor such as silicon or germanium. These inorganic semiconductors have charge carrier mobilities nearly three order of magnitude higher than typical organic semiconductor (Mason et. al, 2002). As a result of this limitation, organic semiconductors are not suitable for use in electronic applications that require very high switching speeds. However, the performance of some organic semiconductors, coupled with their ease of processing makes it competitive in electronic applications that do not require high switching speed such as amorphous silicon. Organic semiconductors can be divided into two types, short chain (oligomers) and long chain (polymers) (Shaw and Seidler, 2001). Typical examples for semiconducting oligomers are pentacene, anthracene, tetracene, and rubrene. Some semiconducting polymers are Poly (3- hexylthiophene) and poly ( $p$-phenylene vinylene).

Short chain organic semiconductors are usually formed by a series of benzene rings in which the nbonds become delocalized to form a n-system. Long chain organic semiconductors are usually polymers that are delocalized along the chain to form a onedimensional system resulting in a 1D-band structure that has considerable band width. The transport properties of such polymers are usually determined by defects in the 1D-chains or by hopping from chain to chain. Polymer organic semiconductors are usually deposited in wet processes, like spin coating or doctor blading.

Tetracene has been identified as an organic semiconductor with chemical formula $\mathrm{C}_{18} \mathrm{H}_{12}$. It is a polycyclic aromatic hydrocarbon. Tetracene is a molecular organic semiconductor, used in organic field-effect transistors (DEFTs) and organic lightemitted diode (OLEDs). In May 2007, researchers from two Japanese universities, Tohoku University in Sendai and Osaka University, reported an ambipolar light-emitting transistor made of a single tetracene crystal (Takashi et al., 2007). Ambipolar means that the electric charge is transported by both positively charged holes and negatively charged electrons. Tetracene can also be used as a gain medium in dye lasers as sensitizer in chemo luminescence. Jan Hendrik Schön during his time at Bell Labs (1997-2002) claimed to have developed an electrically pumped laser based on tetracene. In February 2014, National Aeronautic and space Agency (NASA) announced a greatly upgraded database for tracking polycyclic aromatic hydrocarbons (PAHs), including Tetracene, in the universe (Hoover, 2014). The frontier orbital (LUMO and HOMO) energies of tetracene molecule have been invetigated and reported by (Peter et al., 2009 and Musa et al., 2015).<smiles>c1ccc2cc3cc4ccccc4cc3cc2c1</smiles>

Figure 1. Structure of Tetracence molecule

In this work, geometry optimization and vibrational analysis of tetracene neutral molecule in order to find out the effects in both gas phase and in methanol are intended to be carried out. The effects of different levels of theory and basis sets will also be investigated.

\section{THEORETICAL BACKGROUND}

\section{Density Functional Theory (DFT)}

Density Functional Theory (DFT) is a computational method that derives properties of the molecules based on the determination of their electron density. DFT methods have become the most widely-spread ab-initio methods in Computational Materials Science (CMS) and Solid state Physics, due to their high computational efficiency and very good accuracy for the structure of molecules, crystals, surfaces and their interactions. In DFT methods, the energy of the molecule is a functional of the electron density (Gidado et al., 2015).

\section{Vibrational Frequency}

The vibrational frequencies are calculated with the following equations (Gidado et. al., 2015)

$$
V_{i j}=\frac{1}{\sqrt{m_{i} M_{j}}}\left(\frac{\partial^{n_{2}} V}{\partial_{q i} \partial_{q j}}\right)
$$

where $V_{i j}$ is the Hessian matrix, $m_{\mathrm{i}}$ refers to the mass of atom $I$, and ${ }^{\mathcal{E}}$ al refers to a displacement of atom $i$ in the $\mathrm{x}^{-}, \mathrm{y}^{-}$, or $\mathrm{z}$-direction,

$$
v U=2 U
$$

where $U$ is a matrix of eigenvectors and $\lambda$ is a vector of eigen values, and

$$
\left.\lambda_{k=\left(2-w_{k}\right.}\right)^{z}
$$

Where $\lambda_{k}$ is the $k^{\text {th }}$ eigen value and $v_{k}$ is the $k^{\text {th }}$ vibrational frequency.

The infrared intensities can be computed with the equation (Gidado et al., 2015) 
BAJOPAS Volume 10 Number 1 June, 2017

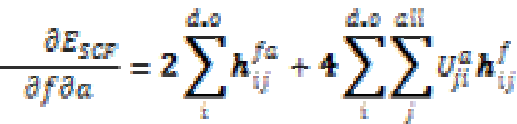

$$
\begin{aligned}
& h_{i j}^{f a}=\sum_{\mu w}^{A 0} C_{p}^{i \theta} C_{w}^{i \theta}\left(\frac{\partial^{2} h_{w w}}{\partial f \partial a}\right)
\end{aligned}
$$

$E_{s c g}$ is the self-consistent field energy, $f$ is the electric field, a is a nuclear coordinate, $\boldsymbol{h}_{\mu v}$ is the one-electron atomic orbital integral, $U^{\ominus}$ is related to the derivative of the molecular orbital coefficients with respect to $a$ by

$$
\frac{\partial c_{\mu}^{i}}{\partial a}=\sum_{m}^{a n l} v_{m}^{a} c_{m}^{m o}
$$

The term "all" in the above summations refers to all occupied and virtual molecular orbitals and .d.o... Refers to doubly occupied orbitals such as those found in the ground state of a closed-shell system.

Terms such as $C_{m}^{i 0}$ refers to the coefficients of the atomic orbital $\mathrm{m}$ in the ith unperturbed molecular orbital.

\section{HOMO-LUMO Energy}

The Highest occupied molecular orbital energy (HOMO) and lowest unoccupied molecular orbital energy (LUMO) are very popular quantum chemical parameters. These orbital's, also called the frontier orbital's, determine the way the molecule interacts with other species. The HOMO is the orbital that could act as an electron donor, since it is the outermost (highest energy) orbital containing electrons. The LUMO is the orbital that could act as the electron acceptor, since it is the innermost (lowest energy) orbital that has room to accept electrons. According to the frontier molecular orbital theory, the formation of a transition state is due to an interaction between the frontier orbital's (HOMO and LUMO) of reactants. The energy of the HOMO is directly related to the ionization potential and the energy of the LUMO is directly related to the electron affinity. The HOMOLUMO gap, that is the difference in energy between the HOMO and LUMO, is an important stability index. A large HOMO-LUMO gap implies high stability for the molecule in chemical reactions. The concept of "activation hardness" has been also defined on the basis of the HOMO-LUMO energy gap. The qualitative definition of hardness is closely related to the polarizability, since a decrease of the energy gap usually leads to easier polarization of the molecule. The HOMO-LUMO gap is defined as (Gidado et. al., 2015)

$\underline{\Delta E}=E_{\text {LMD }}-E_{\text {HOMD }}$

Where

$\Delta \Sigma=$ Is the HOMO-LUMO gap energy

$E_{\text {mOMO }}=$ Is the HOMO energy

$E_{\text {IUMO }}=$ Is the LUMO energy

\section{MATERIALS AND METHODS}

All computations were carried out using Windows Version of Gaussian 03 software (Frisch et al., 2004). The molecular structures and geometries of tetracene have been completely optimized using ab-initio quantum mechanical calculations at the Restricted Hartree-Fock (RHF) and Density Functional Theory (DFT) level of theory without using any symmetry constraints using various basis sets. Gaussian 03 software is a computational Physics and Chemistry program that is used for electronic and geometric structure optimization (geometry optimization, transition states, single point calculation and reaction path modeling), molecular properties, vibrational analysis, electrostatic potential, electron density, and multi-pole moment e.t.c. using both DFT and RHF methods. Geometry optimizations were performed using RHF and DFT levels of theory with different basis sets such as 6-31G, 6-31G (d), 6-31+G and 6$31 \mathrm{G}(\mathrm{d}, \mathrm{p})$. A basis set is a set of wave functions that described the shape of atomic orbital's (AOs). The Density Functional Theory is a cost effective method for inclusion of electron correlations. Geometry optimization is a name for the procedure that attempts to find the configuration of minimum energy of the molecule. The procedure calculated the wave function and the energy at the starting geometry and then proceeded to search a new geometry of a lower energy. This was repeated until the lowest energy geometry was found. In a nut shell geometry optimization was done by locating both the minima and transition states on the potential surface of the molecular orbitals. The optimized molecule obtained from geometry optimization was used as the starting geometry for vibrational calculations. The vibrational analysis was performed by computing the Hessian matrix and the force constants for all the normal modes of the molecule. The frequencies were scaled with a factor of 0.89 in order to remove the effect of the harmonic oscillator. Frequency job began by computing the energy of the input structure. It then went on to compute the frequencies for the structures. Gaussian predicted the frequencies and intensities of each spectral line. The HOMO-LUMO Energy was calculated and from which the energy gap was calculated as the difference in energy between the HOMO and LUMO. IRPal 2.0 was used to interpret the spectra.

\section{RESULTS AND DISCUSSION}

\section{Optimized bond lengths $(\AA)$ of Tetracene}

\section{Molecule in Gas Phase and methano}

The optimized bond lengths of tetracene molecule at two different levels of theory (RHF and B3LYP) in gas phase and Methanol are listed in Tables 1 and 2. The bond length is the measurable distance between two atoms covalently bonded together. It is worth noting that the shorter the bond length the higher is the value of the bond energy (Suzuki e.tal, 2006). The results obtained show that the bond lengths using RHF method has the lowest average value of $1.072 \AA$ and at DFT level has the lowest average value of $1.085 \AA$ in the gas phase. In Methanol, it is observed that at RHF level, the lowest average value was $1.075 \AA$ and at DFT level, it was $1.087 \AA$. 
This indicates that the values are a bit higher in methanol than in gas the phase which implies that the bonds will be slightly stronger in the gas phase than in solution form.

Table1 also shows that the basis set 3-21G gives the lowest values of the bond lengths and 6-31G+ gives the highest values of the bond lengths at RHF level of theory. It is observed that the bonds $R(20,28): C 20-$ $\mathrm{H} 28$ and $\mathrm{R}(23,29): \mathrm{C} 23-\mathrm{H} 29$ follow by $\mathrm{R}(13,21)$ : $\mathrm{C} 23-$ $\mathrm{H} 21$, $\mathrm{R}(14,22): \mathrm{C} 14-\mathrm{H} 22$, and $\mathrm{R}(17,25): \mathrm{C} 17-\mathrm{H} 25$ between carbon and hydrogen atoms at the indicated positions have the lowest values of bond lengths ranging from $1.0717 \AA$ to $1.0728 \AA$. Similarly for B3LYP level of theory, the basis set 3-21G gives the lowest values of the bond lengths and 6-31G+ gives the highest values. It is predicted that the bonds $\mathrm{R}(13,21) ; \mathrm{C} 13-\mathrm{H} 21$ and $\mathrm{R}(14,22): \mathrm{C} 14-\mathrm{H} 22$ follow by $R(7,15)$ and $R(19,27)$ between carbon and hydrogen atoms at the indicated positions possess the lowest values of the bond lengths ranging between $1.0853 \AA$ to $1.085 \AA$. These are the strongest bonds and have the largest values of bond energy in the Tetracene molecule which cannot be easily broken. A large amount of energy is needed to break them. On the other hand, at both levels of theory, the bonds $\mathrm{R}(6,14)$ :C6-C14, R(12,18):C12-C18, R(20,24)C20-C24, and $\mathrm{R}(1,2): \mathrm{C} 1-\mathrm{C} 2, \mathrm{R}(5,11): \mathrm{C} 5-\mathrm{C} 11, \mathrm{R}(6,12)$ :C6-C12 between Carbon-Carbon atoms at the specified positions have the highest values of bond lengths ranging from $1.4425 \AA$ to $1.4557 \AA$. These are the weakest bonds and less amount of energy are required to break them.

Similarly, Table 2 shows that at both levels of theory (RHF and DFT), the basis sets $3-21 \mathrm{G}$ and $3-21 \mathrm{G}+$ give the least values of the bond lengths and $6-31 \mathrm{G}$ gives the highest values. It is predicted that the bonds $R(4$, 10):C4-H10, R (7, 15):C7-H15, R (13, 21):C13-H21 and $\mathrm{R}(17,25): \mathrm{C} 17-\mathrm{H} 25$ between Carbon and Hydrogen atoms at the specified positions have the lowest values of bond lengths ranging from $1.0753 \AA$ to $1.0762 \AA$ at $R H F / 3-21 G$. Similarly the bonds R (24, 30): $\mathrm{C} 24-\mathrm{H} 30, \mathrm{R}$ (23, 29): $\mathrm{C} 23-\mathrm{H} 29$ and R (20, 28): $\mathrm{C} 20-\mathrm{H} 28$ between Carbon and Hydrogen atoms at the indicated positions possess the least values of bond lengths ranging within $1.0863 \AA$ at B3LYP/3$21 \mathrm{G}+$. These are the strongest and hence have the largest values of bond energy in Tetracene molecule which cannot be easily broken. On the other hand, the bonds $\mathrm{R}(5,13)$ : $\mathrm{C} 5-\mathrm{C} 13, \mathrm{R}(11,17)$ :C11-C17, R $(1,2)$ : $\mathrm{C} 1-\mathrm{C} 2$ and $\mathrm{R}(5,11)$ between Carbon-Carbon atoms at the specified positions have the highest values ranging from $1.4441 \AA$ at $\mathrm{RHF}$ to $1.4569 \AA$ at B3LYP. These are the weakest and hence have the lowest values of bond energy. Therefore less amount of energy will be required to break them.

Table 1: Optimized Bond Lengths $(\AA)$ of Tetracene Molecule Gas Phase

\begin{tabular}{|c|c|c|c|c|c|c|c|c|}
\hline \multirow[t]{2}{*}{ Bond lengths } & \multicolumn{4}{|c|}{ RHF } & \multicolumn{4}{|c|}{ B3LYP } \\
\hline & $3-21 G$ & $3-21 \mathrm{G}+$ & $6-31 G$ & $6-31 \mathrm{G}+$ & $3-21 G$ & 3-21G+ & $6-31 G$ & $6-31 \mathrm{G}+$ \\
\hline$R(1,2)$ & 1.4287 & 1.4287 & 1.4309 & 1.4313 & 1.4557 & 1.456 & 1.4563 & 1.4567 \\
\hline$R(1,3)$ & 1.4055 & 1.4055 & 1.4078 & 1.409 & 1.4109 & 1.4125 & 1.413 & 1.4143 \\
\hline$R(1,4)$ & 1.4056 & 1.4056 & 1.4078 & 1.409 & 1.4109 & 1.4125 & 1.413 & 1.4143 \\
\hline $\mathrm{R}(2,7)$ & 1.4055 & 1.4055 & 1.4078 & 1.409 & 1.4109 & 1.4125 & 1.413 & 1.4143 \\
\hline$R(2,8)$ & 1.4056 & 1.4056 & 1.4078 & 1.409 & 1.4109 & 1.4125 & 1.413 & 1.4143 \\
\hline$R(3,6)$ & 1.3722 & 1.3722 & 1.3774 & 1.3787 & 1.3924 & 1.3943 & 1.3961 & 1.3974 \\
\hline$R(3,9)$ & 1.0736 & 1.0736 & 1.0747 & 1.0751 & 1.0858 & 1.0876 & 1.0872 & 1.0877 \\
\hline$R(4,5)$ & 1.3721 & 1.3721 & 1.3773 & 1.3787 & 1.3924 & 1.3943 & 1.3961 & 1.3973 \\
\hline $\mathrm{R}(4,10)$ & 1.0736 & 1.0736 & 1.0747 & 1.0751 & 1.0858 & 1.0876 & 1.0872 & 1.0877 \\
\hline$R(5,11)$ & 1.4373 & 1.4373 & 1.4387 & 1.4393 & 1.4547 & 1.4555 & 1.4556 & 1.4563 \\
\hline$R(5,13)$ & 1.4425 & 1.4425 & 1.4417 & 1.4427 & 1.4363 & 1.4378 & 1.4366 & 1.438 \\
\hline$R(6,12)$ & 1.4372 & 1.4372 & 1.4387 & 1.4393 & 1.4547 & 1.4555 & 1.4556 & 1.4563 \\
\hline$R(6,14)$ & 1.4425 & 1.4425 & 1.4417 & 1.4426 & 1.4363 & 1.4378 & 1.4366 & 1.438 \\
\hline$R(7,12)$ & 1.3722 & 1.3722 & 1.3774 & 1.3787 & 1.3924 & 1.3943 & 1.3961 & 1.3974 \\
\hline$R(7,15)$ & 1.0736 & 1.0736 & 1.0747 & 1.0751 & 1.0858 & 1.0876 & 1.0872 & 1.0877 \\
\hline $\mathrm{R}(8,11)$ & 1.3721 & 1.3721 & 1.3773 & 1.3787 & 1.3924 & 1.3943 & 1.3961 & 1.3973 \\
\hline$R(8,16)$ & 1.0736 & 1.0736 & 1.0747 & 1.0751 & 1.0858 & 1.0876 & 1.0872 & 1.0877 \\
\hline $\mathrm{R}(11,17)$ & 1.4425 & 1.4425 & 1.4417 & 1.4427 & 1.4363 & 1.4378 & 1.4366 & 1.438 \\
\hline $\mathrm{R}(12,18)$ & 1.4425 & 1.4425 & 1.4417 & 1.4426 & 1.4363 & 1.4378 & 1.4366 & 1.438 \\
\hline$R(13,19)$ & 1.3414 & 1.3414 & 1.3479 & 1.3499 & 1.3662 & 1.3692 & 1.3712 & 1.3731 \\
\hline$R(13,21)$ & 1.0728 & 1.0728 & 1.0739 & 1.0742 & 1.085 & 1.0865 & 1.0864 & 1.0869 \\
\hline$R(14,20)$ & 1.3414 & 1.3414 & 1.3479 & 1.35 & 1.3662 & 1.3692 & 1.3712 & 1.3731 \\
\hline$R(14,22)$ & 1.0728 & 1.0728 & 1.0739 & 1.0742 & 1.085 & 1.0865 & 1.0864 & 1.0869 \\
\hline $\mathrm{R}(17,23)$ & 1.3414 & 1.3414 & 1.3479 & 1.3499 & 1.3662 & 1.3692 & 1.3712 & 1.3731 \\
\hline$R(17,25)$ & 1.0728 & 1.0728 & 1.0739 & 1.0742 & 1.085 & 1.0865 & 1.0864 & 1.0869 \\
\hline $\mathrm{R}(18,24)$ & 1.3414 & 1.3414 & 1.3479 & 1.35 & 1.3662 & 1.3692 & 1.3712 & 1.3731 \\
\hline
\end{tabular}


BAJOPAS Volume 10 Number 1 June, 2017

Table 1 continue

\begin{tabular}{lllllllll}
\hline Bond lengths & \multicolumn{3}{c}{ RHF } & \multicolumn{3}{c}{ B3LYP } \\
& $3-21 G$ & $3-21 G+$ & $6-31 G$ & $6-31 G+$ & $3-21 G$ & $3-21 G+$ & $6-31 G$ & $6-31 G+$ \\
$R(18,26)$ & 1.0728 & 1.0728 & 1.0739 & 1.0742 & 1.085 & 1.0865 & 1.0864 & 1.0869 \\
$R(19,23)$ & 1.439 & 1.439 & 1.4376 & 1.4386 & 1.4327 & 1.4348 & 1.433 & 1.4345 \\
$R(19,27)$ & 1.0717 & 1.0717 & 1.0729 & 1.0732 & 1.0838 & 1.0853 & 1.0853 & 1.0857 \\
$R(20,24)$ & 1.439 & 1.439 & 1.4376 & 1.4386 & 1.4327 & 1.4348 & 1.433 & 1.4345 \\
$R(20,28)$ & 1.0717 & 1.0717 & 1.0729 & 1.0732 & 1.0838 & 1.0853 & 1.0853 & 1.0857 \\
$R(23,29)$ & 1.0717 & 1.0717 & 1.0729 & 1.0732 & 1.0838 & 1.0853 & 1.0853 & 1.0857 \\
$R(24,30)$ & 1.0717 & 1.0717 & 1.0729 & 1.0732 & 1.0838 & 1.0853 & 1.0853 & 1.0857 \\
\hline
\end{tabular}

Table 2: Optimized Bond Lengths $(\AA)$ of Tetracene Molecule in Methanol.

\begin{tabular}{|c|c|c|c|c|c|c|c|c|}
\hline \multirow{2}{*}{$\begin{array}{l}\text { Bond } \\
\text { lengths }\end{array}$} & \multicolumn{4}{|c|}{ RHF } & \multicolumn{4}{|c|}{ B3LYP } \\
\hline & 3-21G & $3-21 \mathrm{G}+$ & $6-31 G$ & $6-31 \mathrm{G}+$ & $3-21 G$ & $3-21 \mathrm{G}+$ & $6-31 G$ & $6-31 \mathrm{G}+$ \\
\hline $\mathrm{R}(1,2)$ & 1.4302 & 1.4302 & 1.4322 & 1.4291 & 1.4571 & 1.4571 & 1.0863 & 1.4537 \\
\hline $\mathrm{R}(1,3)$ & 1.407 & 1.407 & 1.4092 & 1.4095 & 1.4124 & 1.4124 & 1.4143 & 1.4115 \\
\hline $\mathrm{R}(1,4)$ & 1.407 & 1.407 & 1.4092 & 1.4095 & 1.4124 & 1.4124 & 1.4143 & 1.4115 \\
\hline $\mathrm{R}(2,7)$ & 1.407 & 1.407 & 1.4092 & 1.4095 & 1.4124 & 1.4124 & 1.4143 & 1.4115 \\
\hline$R(2,8)$ & 1.407 & 1.407 & 1.4092 & 1.375 & 1.4124 & 1.4124 & 1.4143 & 1.4115 \\
\hline$R(3,6)$ & 1.3737 & 1.3737 & 1.3788 & 1.0786 & 1.3939 & 1.3939 & 1.3974 & 1.3939 \\
\hline$R(3,9)$ & 1.0762 & 1.0762 & 1.0769 & 1.3749 & 1.0885 & 1.0885 & 1.0894 & 1.0904 \\
\hline$R(4,5)$ & 1.3737 & 1.3737 & 1.3788 & 1.0786 & 1.3939 & 1.3939 & 1.3974 & 1.3939 \\
\hline$R(4,10)$ & 1.0762 & 1.0762 & 1.0769 & 1.4395 & 1.0885 & 1.0885 & 1.0894 & 1.0904 \\
\hline$R(5,11)$ & 1.4388 & 1.4388 & 1.4401 & 1.4457 & 1.4562 & 1.4562 & 1.4569 & 1.4532 \\
\hline$R(5,13)$ & 1.4441 & 1.4441 & 1.4431 & 1.4395 & 1.4379 & 1.4379 & 1.4381 & 1.4353 \\
\hline$R(6,12)$ & 1.4388 & 1.4388 & 1.44 & 1.4457 & 1.4562 & 1.4562 & 1.4569 & 1.4532 \\
\hline$R(6,14)$ & 1.444 & 1.444 & 1.4431 & 1.375 & 1.4379 & 1.4379 & 1.4381 & 1.4353 \\
\hline$R(7,12)$ & 1.3737 & 1.3737 & 1.3788 & 1.0786 & 1.3939 & 1.3939 & 1.3974 & 1.3939 \\
\hline$R(7,15)$ & 1.0762 & 1.0762 & 1.0769 & 1.3749 & 1.0885 & 1.0885 & 1.0894 & 1.0904 \\
\hline $\mathrm{R}(8,11)$ & 1.3737 & 1.3737 & 1.3788 & 1.0786 & 1.3939 & 1.3939 & 1.3974 & 1.3939 \\
\hline$R(8,16)$ & 1.0762 & 1.0762 & 1.0769 & 1.4457 & 1.0885 & 1.0885 & 1.0894 & 1.0904 \\
\hline $\mathrm{R}(11,17)$ & 1.4441 & 1.4441 & 1.4431 & 1.4457 & 1.4379 & 1.4379 & 1.4381 & 1.4353 \\
\hline$R(12,18)$ & 1.444 & 1.444 & 1.4431 & 1.3437 & 1.4379 & 1.4379 & 1.4381 & 1.4353 \\
\hline$R(13,19)$ & 1.3429 & 1.3429 & 1.3494 & 1.078 & 1.3677 & 1.3677 & 1.3726 & 1.3683 \\
\hline$R(13,21)$ & 1.0753 & 1.0753 & 1.0762 & 1.3438 & 1.0876 & 1.0876 & 1.0887 & 1.0898 \\
\hline$R(14,20)$ & 1.3429 & 1.3429 & 1.3494 & 1.078 & 1.3677 & 1.3677 & 1.3726 & 1.3683 \\
\hline$R(14,22)$ & 1.0753 & 1.0753 & 1.0762 & 1.3437 & 1.0876 & 1.0876 & 1.0887 & 1.0898 \\
\hline$R(17,23)$ & 1.3429 & 1.3429 & 1.3494 & 1.3437 & 1.3677 & 1.3677 & 1.3726 & 1.3683 \\
\hline$R(17,25)$ & 1.0753 & 1.0753 & 1.0762 & 1.078 & 1.0876 & 1.0876 & 1.0887 & 1.0898 \\
\hline$R(18,24)$ & 1.3429 & 1.3429 & 1.3494 & 1.3438 & 1.3677 & 1.3677 & 1.3726 & 1.3683 \\
\hline$R(18,26)$ & 1.0753 & 1.0753 & 1.0762 & 1.078 & 1.0876 & 1.0876 & 1.0887 & 1.0898 \\
\hline$R(19,23)$ & 1.4408 & 1.4408 & 1.4393 & 1.4435 & 1.4345 & 1.4345 & 1.4347 & 1.4323 \\
\hline$R(19,27)$ & 1.0742 & 1.0742 & 1.0751 & 1.0773 & 1.0863 & 1.0863 & 1.0876 & 1.0888 \\
\hline$R(20,24)$ & 1.4407 & 1.4407 & 1.4393 & 1.4434 & 1.4345 & 1.4345 & 1.4346 & 1.4323 \\
\hline$R(20,28)$ & 1.0742 & 1.0742 & 1.0751 & 1.0773 & 1.0863 & 1.0863 & 1.0876 & 1.0888 \\
\hline$R(23,29)$ & 1.0742 & 1.0742 & 1.0751 & 1.0773 & 1.0863 & 1.0863 & 1.0876 & 1.0888 \\
\hline$R(24,30)$ & 1.0742 & 1.0742 & 1.0751 & 1.0773 & 1.0863 & 1.0863 & 1.0876 & 1.0888 \\
\hline
\end{tabular}

Optimized bond angle $(\AA)$ of Tetracene molecule in gas phase and in methanol

The optimized bond angles of tetracene molecule at both levels of theory (RHF and B3LYP) in gas phase and methanol are listed in Table 3 and 4 . The bond angle is the average angle between the orbitals of the central atom containing the bonding electron pairs in the molecule (Mason and Brady, 2007). It is expressed in degrees. The bond angle throws more light on the distribution of orbital's around a central atom in a molecule. The bond angles also contribute to the shape of a molecule. 
It is observed that all the bond angles in Tetracene molecule are so closed to $120^{\circ}$ in both gas phase and methanol suggesting that the molecule is planar benzene in which the $C$ atom are $s p^{2}$ hybridized. In gas phase, the angles with least values include; $A(12,18,26): C 12-C 18-H 26, \quad A(5,13,21): C 5-C 13-H 21$ and $A(11,17,25): C 5-C 17-\mathrm{H} 25$ with the values of $118.04^{\circ}$ at RHF/3-21G and $118.357^{\circ}$ at B3LYP/6-31G+ respectively. These are all $\mathrm{C}-\mathrm{C}-\mathrm{H}$ angles. But C-C-C angles: $\quad A(11,17,23): C 11-C 17-C 23, \quad A(11,5,13): C 11-$ $C 5-C 13$ and $A(5,11,17): C 5-C 11 C 17$ have values within $118.041^{\circ}$ to $118.4556^{\circ}$ at both levels of theory. In methanol, the angles with the lowest values are $\mathrm{A}(12,18,26): \mathrm{C} 12-\mathrm{C} 18-\mathrm{H} 26, \quad \mathrm{~A}(5,13,21): \mathrm{C} 5-\mathrm{C} 13-\mathrm{H} 21$, $A(11,17,25): C 11-C 17-H 25$ and $A(6,14,22): C 6-C 14-$ $\mathrm{H} 22$ with the values ranging within $118.023^{\circ}$ to $118.3218^{\circ}$ at both levels of theory. These are all C-C$\mathrm{H}$ angles. The $\mathrm{C}-\mathrm{C}-\mathrm{C}$ angles are; A $(12,6,14), \mathrm{A}(11$, $5,13)$ and $A(5,11,17)$ with bond angles of $118.3891^{\circ}, 118.4824^{\circ}$ and $118.3885^{\circ}$ respectively. This also shows that the values obtained in solution are a bit higher than the one obtained in gas phase by about $0.09^{\circ}$.

Table 3: Optimized Bond Angles $(\AA)$ of Tetracene molecule in Gas.

\begin{tabular}{|c|c|c|c|c|c|c|c|c|}
\hline Bond Angles & $\begin{array}{l}\text { RHF } \\
\text { 3-21G }\end{array}$ & 3-21G+ & $6-31 G$ & $6-31 \mathrm{G}+$ & $\begin{array}{l}\text { B3LYP } \\
\text { 3-21G }\end{array}$ & $3-21 G+$ & $6-31 G$ & $6-31 \mathrm{G}+$ \\
\hline$A(2,1,3)$ & 118.9134 & 118.9134 & 118.8889 & 118.9009 & 118.8693 & 118.8897 & 118.8349 & 118.8444 \\
\hline$A(2,1,4)$ & 118.9127 & 118.9127 & 118.8884 & 118.9004 & 118.8693 & 118.8898 & 118.835 & 118.8445 \\
\hline$A(2,1,4)$ & 122.1739 & 122.1739 & 122.2227 & 122.1987 & 122.2614 & 122.2204 & 122.3301 & 122.3112 \\
\hline$A(1,2,7)$ & 118.9134 & 118.9134 & 118.8889 & 118.9009 & 118.8695 & 118.89 & 118.8351 & 118.8446 \\
\hline$A(1,2,8)$ & 118.9126 & 118.9126 & 118.8884 & 118.9004 & 118.8691 & 118.8896 & 118.8348 & 118.8443 \\
\hline$A(7,2,8)$ & 122.1739 & 122.1739 & 122.2227 & 122.1987 & 122.2614 & 122.2204 & 122.3301 & 122.3112 \\
\hline$A(1,3,6)$ & 121.6061 & 121.6061 & 121.7074 & 121.6912 & 121.8176 & 121.7958 & 121.9307 & 121.9169 \\
\hline$A(1,3,9)$ & 118.8061 & 118.8061 & 118.8063 & 118.8193 & 118.8519 & 118.8643 & 118.8139 & 118.8293 \\
\hline$A(6,3,9)$ & 119.5878 & 119.5878 & 119.4863 & 119.4895 & 119.3305 & 119.3399 & 119.2553 & 119.2538 \\
\hline$A(1,4,5)$ & 121.6066 & 121.6066 & 121.7077 & 121.6916 & 121.8177 & 121.7959 & 121.9308 & 121.917 \\
\hline $\mathrm{A}(1,4,10)$ & 118.8045 & 118.8045 & 118.805 & 118.818 & 118.8518 & 118.8644 & 118.8138 & 118.8292 \\
\hline$A(5,4,10)$ & 119.589 & 119.589 & 119.4873 & 119.4905 & 119.3305 & 119.3396 & 119.2554 & 119.2539 \\
\hline $\mathrm{A}(4,5,11)$ & 119.4808 & 119.4808 & 119.4039 & 119.4081 & 119.3131 & 119.3143 & 119.2343 & 119.2386 \\
\hline $\mathrm{A}(4,5,13)$ & 122.1948 & 122.1948 & 122.2325 & 122.209 & 122.2637 & 122.2328 & 122.31 & 122.2964 \\
\hline$A(11,5,13)$ & 118.3243 & 118.3243 & 118.3636 & 118.3829 & 118.4232 & 118.4529 & 118.4558 & 118.465 \\
\hline$A(3,6,12)$ & 119.4805 & 119.4805 & 119.4037 & 119.4079 & 119.313 & 119.3143 & 119.2343 & 119.2386 \\
\hline$A(3,6,14)$ & 122.1945 & 122.1945 & 122.2322 & 122.2087 & 122.2637 & 122.2328 & 122.31 & 122.2963 \\
\hline$A(12,6,14)$ & 118.3251 & 118.3251 & 118.3641 & 118.3834 & 118.4233 & 118.4529 & 118.4558 & 118.465 \\
\hline$A(2,7,12)$ & 121.6062 & 121.6062 & 121.7075 & 121.6913 & 121.8176 & 121.7958 & 121.9307 & 121.9169 \\
\hline$A(2,7,15)$ & 118.8062 & 118.8062 & 118.8064 & 118.8193 & 118.8519 & 543 & 118 & 8293 \\
\hline$A(12,7,15)$ & 119.5876 & 119.5876 & 119.4861 & 119 & 119.3305 & 398 & 553 & 2538 \\
\hline $\mathrm{A}(2,8,11)$ & 121.6064 & 121.6064 & 121.7077 & 121.6915 & 121.8177 & 121.7959 & 308 & 169 \\
\hline $\mathrm{A}(2,8,16)$ & 118.8045 & 118.8045 & 118.8051 & 118.818 & 118.8514 & 118.8639 & 118.8135 & 118.8288 \\
\hline$A(11,8,16)$ & 119.5891 & 119.5891 & 119.4873 & 119.4905 & 119.3309 & 119.3402 & 119.2557 & 119.2542 \\
\hline$A(5,11,8)$ & 119.4808 & 119.4808 & 119.4039 & 119.4081 & 119.3131 & 119.3144 & 119.2344 & 119.2388 \\
\hline$A(5,11,17)$ & 118.3244 & $11 \varepsilon$ & 536 & & 11 & 527 & 556 & 448 \\
\hline $\mathrm{A}(8,11,17)$ & 122.1948 & 122.1948 & 122.2324 & 122.209 & 122.2638 & 122.2329 & 122.31 & 122.2964 \\
\hline$A(6,12,7)$ & 119.4804 & 119.4804 & 119.4036 & 119.4078 & 119.313 & 119.3143 & 343 & 119.2386 \\
\hline$A(6,12,18)$ & 118.3251 & 118.3251 & 118.3642 & 118.3835 & 118.4233 & 118.4529 & 118.4558 & 118.465 \\
\hline $\mathrm{A}(7,12,18)$ & 122.1945 & 122.1945 & 122.2322 & 122.2087 & 122.2637 & 122.2328 & 122.31 & 122.2963 \\
\hline$A(5,13,19)$ & 121.039 & 121. & 121.0706 & 121.0684 & 121.0162 & 121.0259 & 121.0513 & 121.0644 \\
\hline$A(5,13,21)$ & 118.0398 & 118.0398 & 118.2163 & 118.2557 & 118.2282 & 118.2967 & 118.3131 & 118.357 \\
\hline $\mathrm{A}(19,13,21)$ & 120.9212 & 120.9212 & 120.7131 & 120 & 120.7556 & 120.6774 & 120.6356 & 5786 \\
\hline$A(6,14,20)$ & 121.0385 & 121.0385 & 121.0702 & 121.068 & 121.0161 & 121.0258 & 121.0512 & 121.0643 \\
\hline$A(6,14,22)$ & 118.041 & 118.041 & 118.2171 & 118.2566 & 118.2286 & 118.2971 & 118.3135 & 118.3574 \\
\hline$A(20,14,22)$ & 120.9206 & & 120.7126 & & 120.7553 & 120.677 & .6353 & 120.5783 \\
\hline $\mathrm{A}(11,17,23)$ & 121.0388 & 121.0 & 121.0705 & 82 & 162 & 259 & 513 & 0644 \\
\hline$A(11,17,25)$ & 118.0399 & 118.0399 & 118.2163 & 118.2557 & 118.2282 & 118.2967 & 118.3131 & 118.357 \\
\hline$A(23,17,25)$ & 120.9214 & 120.9214 & 120.7133 & 120.6761 & 120.7556 & 120.6774 & 120.6356 & 120.5786 \\
\hline$A(12,18,24)$ & 121.0384 & 121.0384 & 121.0702 & 121.068 & 121.0161 & 121.0258 & 121.0512 & 121.0643 \\
\hline $\mathrm{A}(12,18,26)$ & 118.041 & 118.041 & 118.2171 & 118.2566 & 118.2286 & 118.2971 & 118.3134 & 118.3573 \\
\hline$A(24,18,26)$ & 120.9206 & & & & & & 120.6353 & 120.5783 \\
\hline$A(13,19,23)$ & 120.6367 & 120.6367 & 120.5658 & 120.5487 & 120.5606 & 120.5213 & 120.493 & 120.4706 \\
\hline $\mathrm{A}(13,19,27)$ & 120.5385 & 120.5385 & 120.4621 & 120.4482 & 120.3243 & 120.329 & 120.2655 & 120.2521 \\
\hline $\mathrm{A}(23,19,27)$ & 118.8248 & 118.8248 & 118.9722 & 119.0031 & 119.1151 & 119.1497 & 119.2415 & 119.2773 \\
\hline$A(14,20,24)$ & 120.6365 & 120.6365 & 120.5656 & 120.5485 & 120.5607 & 120.5213 & 120.493 & 120.4706 \\
\hline$A(14,20,28)$ & 120.5378 & 120.5378 & 120.4615 & 120.4477 & 120.3239 & 120.3286 & 120.2652 & 120.2517 \\
\hline $\mathrm{A}(24,20,28)$ & 118.8257 & 118.8257 & 118.9729 & 119.0038 & 119.1155 & 119.1501 & 119.2418 & 119.2776 \\
\hline $\mathrm{A}(17,23,19)$ & 120.6368 & 120.6368 & 120.5659 & 120.5488 & 120.5607 & 120.5214 & 120.4931 & 120.4707 \\
\hline$A(17,23,29)$ & 120.5385 & 120.5385 & 120.462 & 120.4482 & 120.3243 & 120.329 & 120.2655 & 120.2521 \\
\hline$A(19,23,29)$ & 118.8247 & 118.8247 & 118.9721 & 119.003 & 119.115 & 119.1496 & 119.2414 & 119.2773 \\
\hline $\mathrm{A}(18,24,20)$ & 120.6365 & 120.6365 & 120.5656 & 120.5485 & 120.5607 & 120.5213 & 120.493 & 120.4706 \\
\hline $\mathrm{A}(18,24,30)$ & 120.5378 & 120.5378 & 120.4615 & 120.4477 & 120.3239 & 120.3286 & 120.2652 & 120.2517 \\
\hline $\mathrm{A}(20,24,30)$ & 118.8257 & 118.8257 & 118.9729 & 119.0038 & 119.1154 & 119.1501 & 119.2418 & 119.2776 \\
\hline
\end{tabular}


Table 4: Optimized Bond Angles $\left({ }^{0}\right)$ of Tetracene molecule (Methanol)

\begin{tabular}{|c|c|c|c|c|c|c|c|c|}
\hline $\begin{array}{l}\text { Bond } \\
\text { Angles }\end{array}$ & $\begin{array}{l}\text { RHF } \\
\text { 3-21G }\end{array}$ & 3-1G+ & 6-31G & 6-1G+ & $\begin{array}{l}\text { B3LYP } \\
\text { 3-21G }\end{array}$ & 3-1G+ & 6-31G & $6-31 G+$ \\
\hline$A(2,1,3)$ & 119.0126 & 119.0126 & 118.9767 & 118.9101 & 118.9604 & 118.9604 & 118.9134 & 118.9101 \\
\hline$A(2,1,4)$ & 119.0119 & 119.0119 & 118.9762 & 118.9101 & 118.9605 & 118.9605 & 118.9135 & 118.9101 \\
\hline$A(2,1,4)$ & 121.9756 & 121.9756 & 122.0471 & 122.1798 & 122.0791 & 122.0791 & 122.1731 & 122.1798 \\
\hline$A(1,2,7)$ & 119.0125 & 119.0125 & 118.9767 & 118.9102 & 118.9606 & 118.9606 & 118.9136 & 118.9102 \\
\hline$A(1,2,8)$ & 119.0118 & 119.0118 & 118.9762 & 118.9099 & 118.9603 & 118.9603 & 118.9133 & 118.9099 \\
\hline$A(7,2,8)$ & 121.9757 & 121.9757 & 122.0471 & 122.1798 & 122.0791 & 122.0791 & 122.1732 & 122.1798 \\
\hline$A(1,3,6)$ & 121.4083 & 121.4083 & 121.5329 & 121.768 & 121.6356 & 121.6356 & 121.7746 & 121.768 \\
\hline$A(1,3,9)$ & 118.8908 & 118.8908 & 118.8832 & 118.8958 & 118.9368 & 118.9368 & 118.8892 & 118.8958 \\
\hline$A(6,3,9)$ & 119.7009 & 119.7009 & 119.5839 & 119.3362 & 119.4277 & 119.4277 & 119.3362 & 119.3362 \\
\hline$A(1,4,5)$ & 121.4088 & 121.4088 & 121.5332 & 121.768 & 121.6357 & 121.6357 & 121.7746 & 121.768 \\
\hline$A(1,4,10)$ & 118.8891 & 118.8891 & 118.8818 & 118.8957 & 118.9366 & 118.9366 & 118.8891 & 118.8957 \\
\hline $\mathrm{A}(5,4,10)$ & 119.7021 & 119.7021 & 119.585 & 119.3363 & 119.4277 & 119.4277 & 119.3363 & 119.3363 \\
\hline$A(4,5,11)$ & 119.5794 & 119.5794 & 119.4906 & 119.3219 & 119.4039 & 119.4039 & 119.3119 & 119.3219 \\
\hline$A(4,5,13)$ & 122.0321 & 122.0321 & 122.0908 & 122.1675 & 122.1137 & 122.1137 & 122.1831 & 122.1675 \\
\hline$A(11,5,13)$ & 118.3884 & 118.3884 & 118.4186 & 118.5107 & 118.4824 & 118.4824 & 118.505 & 118.5107 \\
\hline$A(3,6,12)$ & 119.5791 & 119.5791 & 119.4904 & 119.3219 & 119.4039 & 119.4039 & 119.3119 & 119.3219 \\
\hline$A(3,6,14)$ & 122.0317 & 122.0317 & 122.0905 & 122.1674 & 122.1137 & 122.1137 & 122.183 & 122.1674 \\
\hline$A(12,6,14)$ & 118.3891 & 118.3891 & 118.4191 & 118.5107 & 118.4824 & 118.4824 & 118.5051 & 118.5107 \\
\hline$A(2,7,12)$ & 121.4084 & 121.4084 & 121.533 & 121.768 & 121.6356 & 121.6356 & 121.7746 & 121.768 \\
\hline$A(2,7,15)$ & 118.8908 & 118.8908 & 118.8832 & 118.8958 & 118.9367 & 118.9367 & 118.8892 & 118.8958 \\
\hline$A(12,7,15)$ & 119.7007 & 119.7007 & 119.5838 & 119.3362 & 119.4277 & 119.4277 & 119.3363 & 119.3362 \\
\hline $\mathrm{A}(2,8,11)$ & 121.4087 & 121.4087 & 121.5332 & 121.768 & 121.6356 & 121.6356 & 121.7746 & 121.768 \\
\hline$A(2,8,16)$ & 118.8891 & 118.8891 & 118.8819 & 118.8954 & 118.9362 & 118.9362 & 118.8887 & 118.8954 \\
\hline $\mathrm{A}(11,8,16)$ & 119.7022 & 119.7022 & 119.5849 & 119.3366 & 119.4281 & 119.4281 & 119.3366 & 119.3366 \\
\hline$A(5,11,8)$ & 119.5794 & 119.5794 & 119.4906 & 119.322 & 119.404 & 119.404 & 119.3121 & 119.322 \\
\hline$A(5,11,17)$ & 118.3885 & 118.3885 & 118.4186 & 118.5105 & 118.4822 & 118.4822 & 118.5049 & 118.5105 \\
\hline $\mathrm{A}(8,11,17)$ & 122.0321 & 122.0321 & 122.0908 & 122.1675 & 122.1137 & 122.1137 & 122.1831 & 122.1675 \\
\hline$A(6,12,7)$ & 119.579 & 119.579 & 119.4903 & 119.3219 & 119.4039 & 119.4039 & 119.3119 & 119.3219 \\
\hline$A(6,12,18)$ & 118.3892 & 118.3892 & 118.4192 & 118.5107 & 118.4825 & 118.4825 & 118.5051 & 118.5107 \\
\hline $\mathrm{A}(7,12,18)$ & 122.0318 & 122.0318 & 122.0905 & 122.1674 & 122.1137 & 122.1137 & 122.183 & 122.1674 \\
\hline $\mathrm{A}(5,13,19)$ & 120.9121 & 120.9121 & 120.9672 & 120.9383 & 120.8975 & 120.8975 & 120.9576 & 120.9383 \\
\hline$A(5,13,21)$ & 118.023 & 118.023 & 118.2043 & 118.3334 & 118.235 & 118.235 & 118.3214 & 118.3334 \\
\hline$A(19,13,21)$ & 121.0649 & 121.0649 & 120.8285 & 120.7283 & 120.8675 & 120.8675 & 120.7211 & 120.7283 \\
\hline$A(6,14,20)$ & 120.9117 & 120.9117 & 120.9668 & 120.9382 & 120.8974 & 120.8974 & 120.9574 & 120.9382 \\
\hline$A(6,14,22)$ & 118.0242 & 118.0242 & 118.2052 & 118.3338 & 118.2355 & 118.2355 & 118 & $11 \varepsilon$ \\
\hline $\mathrm{A}(20,14,22)$ & 121.0642 & 121.0642 & 120.828 & 120.728 & 120.8671 & 120.8671 & 120.7208 & 120.728 \\
\hline$A(11,17,23)$ & 120.9119 & 120.9119 & 120.967 & 120.9383 & 120.8974 & 120.8974 & 120.9576 & 120.9383 \\
\hline$A(11,17,25)$ & 118.0231 & 118.0231 & 118.2044 & 118.3335 & 118.235 & 118.235 & 118.3214 & 118.3335 \\
\hline$A(23,17,25)$ & 121.065 & 121.065 & 120.8286 & 120.7283 & 120.8675 & 120.8675 & 120.721 & 120.7283 \\
\hline $\mathrm{A}(12,18,24)$ & 120.9116 & 120.9116 & 120.9668 & 120.9381 & 120.8974 & 120.8974 & 120.9574 & 120.9381 \\
\hline$A(12,18,26)$ & 118.0242 & 118.0242 & 118.2053 & 118.3339 & 118.2354 & 118.2354 & 118.3218 & 118.3339 \\
\hline $\mathrm{A}(24,18,26)$ & 121.0642 & 121.0642 & 120.828 & 120.728 & 120.8672 & 120.8672 & 120.7208 & 120.728 \\
\hline$A(13,19,23)$ & 120.6995 & 120.6995 & 120.6142 & 120.5511 & 120.6202 & 120.6202 & 120.5375 & 120.5511 \\
\hline$A(13,19,27)$ & 120.5259 & 120.5259 & 120.463 & 120.1846 & 120.3029 & 120.3029 & 120.2586 & 120.1846 \\
\hline $\mathrm{A}(23,19,27)$ & 118.7747 & 118.7747 & 118.9228 & 119.2643 & 119.0769 & 119.0769 & 119.2039 & 119.2643 \\
\hline$A(14,20,24)$ & 120.6992 & 120.6992 & 120.6141 & 120.5511 & 120.6202 & 120.6202 & 120.5375 & 120.5511 \\
\hline$A(14,20,28)$ & 120.5251 & 120.5251 & 120.4623 & 120.1842 & 120.3025 & 120.3025 & 120.2583 & 120.1842 \\
\hline$A(24,20,28)$ & 118.7757 & 118.7757 & 118.9236 & 119.2646 & 119.0773 & 119.0773 & 119.2043 & 119.2646 \\
\hline$A(17,23,19)$ & 120.6996 & 120.6996 & 120.6143 & 120.5511 & 120.6203 & 120.6203 & 120.5375 & 120.5511 \\
\hline$A(17,23,29)$ & 120.5258 & 120.5258 & 120.4629 & 120.1846 & 120.3029 & 120.3029 & 120.2586 & 120.1846 \\
\hline$A(19,23,29)$ & 118.7746 & 118.7746 & 118.9228 & 119.2643 & 119.0768 & 119.0768 & 119.2039 & 119.2643 \\
\hline $\mathrm{A}(18,24,20)$ & 120.6992 & 120.6992 & 120.6141 & 120.5511 & 120.6201 & 120.6201 & 120.5375 & 120.5511 \\
\hline$A(18,24,30)$ & 120.5251 & 120.5251 & 120.4623 & 120.1842 & 120.3025 & 120.3025 & 120.2583 & 120.1842 \\
\hline$A(20,24,30)$ & 118.7757 & 118.7757 & 118.9236 & 119.2646 & 119.0773 & 119.0773 & 119.2042 & 119.2646 \\
\hline
\end{tabular}




\section{HOMO-LUMO Energy Gap}

Tables 5 and 6 , show the calculated values of the HOMO, LUMO and HOMO-LUMO Energy gap in atomic unit (a.u) and in electron volt (eV) of the studied molecule in gas phase and in solution. It is clear from Table 5 and 6 that the HOMO-LUMO energy gap for the Tetracene molecule is approximately the same for all the basis sets at RHF level of the theory. At this theory, the energy gap is about $6.77864 \mathrm{eV}$ for the entire basis sets used. But it is observed that at B3LYP level of theory the energy gap for all basis sets used is about $2.456 \mathrm{eV}$ for both gas and methanol. This value is more in agreement to a reported experimental value of 2.63 (Musa et al., 2015). Thus, B3LYP calculations are more in agreement with the experimental value due to the inclusion of electron correlation than RHF. However, the average value of HOMO-LUMO energy gap in gas phase is $2.456 \mathrm{eV}$ which is slightly higher than the average value obtained in solution $2.455 \mathrm{eV}$. This indicates that tetracene is slightly more stable in the gas phase than in solution form.

\begin{tabular}{|c|c|c|c|c|c|c|}
\hline Method & HOMO(a.u) & LUMO(a.u) & & $\begin{array}{l}\text { HOMO- } \\
\text { LUMO Energy } \\
\text { Gap (a.u) }\end{array}$ & $\begin{array}{l}\text { HOMO- } \\
\text { LUMO Energy } \\
\text { Gap(eV) }\end{array}$ & $\begin{array}{c}\text { HOMO- } \\
\text { LUMO } \\
\text { Energy Gap } \\
\text { (eV) (Musa } \\
\text { et al., 2015). }\end{array}$ \\
\hline \multirow[t]{4}{*}{ RHF } & $3-21 G$ & -0.22449 & 0.02498 & 0.24947 & 6.78857764 & \\
\hline & $3-21 G+$ & -0.22449 & 0.02498 & 0.24947 & 6.78857764 & \\
\hline & $6-31 G$ & -0.22005 & 0.02788 & 0.24793 & 6.74667116 & \\
\hline & $6-31 G+$ & -0.21834 & 0.02917 & 0.24751 & 6.73524212 & \\
\hline \multirow[t]{4}{*}{ B3LYP } & $3-21 G$ & -0.17503 & -0.08400 & 0.09103 & 2.4771836 & \\
\hline & $3-21 G+$ & -0.17503 & -0.08400 & 0.09103 & 2.4771836 & 2.63 \\
\hline & $6-31 G$ & -0.17198 & -0.08159 & 0.09039 & 2.4596268 & \\
\hline & $6-31 G+$ & -0.17096 & -0.08239 & 0.08857 & 2.41016684 & \\
\hline
\end{tabular}

\begin{tabular}{|c|c|c|c|c|c|c|}
\hline Method & & & $\begin{array}{l}\text { LUMO } \\
\text { (a.u) }\end{array}$ & $\begin{array}{l}\text { HOMO-LUMO } \\
\text { Energy Gap } \\
\text { (a.u) }\end{array}$ & $\begin{array}{l}\text { HOMO-LUMO } \\
\text { Energy } \\
\text { Gap(eV) }\end{array}$ & $\begin{array}{l}\text { HOMO- LUMO Energy } \\
\text { Gap (eV) (Musa et. } \\
\text { al., 2015). }\end{array}$ \\
\hline \multirow[t]{4}{*}{ RHF } & $3-21 G$ & -0.22781 & 0.02125 & 0.24906 & 6.77742072 & \\
\hline & $3-21 \mathrm{G}+$ & -0.22781 & 0.02125 & 0.24906 & 6.77742072 & \\
\hline & $6-31 G$ & -0.22172 & 0.02610 & 0.24782 & 6.74367784 & \\
\hline & $6-31 G+$ & -0.22004 & 0.02742 & 0.24746 & 6.73388152 & \\
\hline \multirow[t]{4}{*}{ DFT } & $3-21 G$ & -0.17800 & -0.08701 & 0.09099 & 2.47601988 & 2.63 \\
\hline & $3-21 G+$ & -0.17800 & -0.08701 & 0.09099 & 2.47601988 & \\
\hline & $6-31 G$ & -0.17288 & -0.08251 & 0.09037 & 2.45914844 & \\
\hline & $6-31 \mathrm{G}+$ & -0.17175 & -0.08319 & 0.08856 & 2.40989472 & \\
\hline
\end{tabular}

\section{Mulliken Atomic Charges}

The calculation of effective atomic charges plays an important role in the application of quantum mechanical calculations to molecular system because atomic charges affect dipole moment, molecular polarization, electronic structure, and a lot of properties of molecular system (Ciolowski et al., 1998). The charge distribution over the atoms suggests the formation of donor and acceptor pairs involving the charge transfer in the molecule. Atomic charge has been used to describe the processes of electro negativity equalization and charge transfer in chemical reaction (Maksic and Jug, 1991). The interest here is in the comparison of different methods to describe the electron distribution in tetracene molecule as broadly as possible, and assess the sensitivity of the calculated charges to changes in (i) the choice of the basis set; (ii) the choice of the quantum mechanical method. Mulliken charges were calculated by determining the electron population of each atom as defined in the basic functions. The mulliken charges calculated for both gas phase and methanol are listed in Table 7. The data presented clearly shows that mulliken charges are very sensitive to basis set used in calculation. It is worthy to mention that in the basis set 3-21G, all the hydrogen atoms of the title molecule exhibited positive charge values at RHF level of the theory in the gas phase while all carbon atoms exhibited negative charge values. $\mathrm{H} 25$ atom exhibits the highest positive charge value of 0.240669 in the gas phase while in methanol $\mathrm{H} 15$ atom exhibits the highest positive value of 0.283561 at RHF/3-21G. On the other hand, the maximum negative charge value of -0.267558 for $\mathrm{C} 19$ at RHF/3-21G. In methanol, it is observed that all the hydrogen atoms are positive charge values at RHF/3$21 \mathrm{G}$ level of theory. But at B3LYP/6-31G+, all the hydrogen atoms also have positive charge values while all the carbon atoms exhibited negative charge values with the exception of $\mathrm{C5}-\mathrm{C} 6$ which possessed positive charge values of about 0.150176 and 0.149767 respectively. However, the presence of large negative charge value on $\mathrm{C}$ atom and large positive charge value on $\mathrm{H}$ atom may suggest the formation of intra molecular interaction in both forms (Rubarani and Sampath, 2013). Hence, it is also observed that the mulliken charges values in methanol are a bit higher in solution than in gas phase. 
BAJOPAS Volume 10 Number 1 June, 2017

Table 7: Mulliken Atomic charge Distributions in Methanol and in Gas

\begin{tabular}{|c|c|c|}
\hline Atoms & $\begin{array}{l}\text { RHF } \\
3-21 G \\
\text { Charge ( statC) }\end{array}$ & $\begin{array}{l}\text { B3LYP } \\
6-31 G_{+} \\
\text {Charge (statC) }\end{array}$ \\
\hline $\mathrm{C} 1$ & -0.120810 & 0.163157 \\
\hline $\mathrm{C} 2$ & -0.121025 & 0.163629 \\
\hline C3 & -0.171091 & -0.326680 \\
\hline C4 & -0.170889 & -0.326603 \\
\hline C5 & -0.120281 & 0.150176 \\
\hline C6 & -0.120133 & 0.149767 \\
\hline C7 & -0.170918 & -0.326610 \\
\hline $\mathrm{C} 8$ & -0.170922 & -0.326652 \\
\hline H9 & 0.283547 & 0.165004 \\
\hline $\mathrm{H} 10$ & 0.283555 & 0.165010 \\
\hline C11 & -0.120067 & 0.149755 \\
\hline C12 & -0.120138 & 0.149721 \\
\hline C13 & -0.217793 & -0.229758 \\
\hline C14 & -0.217949 & -0.229811 \\
\hline H15 & 0.283561 & 0.165011 \\
\hline H16 & 0.283558 & 0.165011 \\
\hline C17 & -0.217943 & -0.229840 \\
\hline C18 & -0.217949 & -0.229801 \\
\hline C19 & -0.267558 & -0.161363 \\
\hline $\mathrm{C} 20$ & -0.267527 & -0.161319 \\
\hline $\mathrm{H} 21$ & 0.277569 & 0.161674 \\
\hline $\mathrm{H} 22$ & 0.277567 & 0.161673 \\
\hline $\mathrm{C} 23$ & -0.267550 & -0.161323 \\
\hline $\mathrm{C} 24$ & -0.267531 & -0.161326 \\
\hline $\mathrm{H} 25$ & 0.277561 & 0.161669 \\
\hline $\mathrm{H} 26$ & 0.277567 & 0.161672 \\
\hline $\mathrm{H} 27$ & 0.275886 & 0.159531 \\
\hline $\mathrm{H} 28$ & 0.275904 & 0.159545 \\
\hline $\mathrm{H} 29$ & 0.275898 & 0.159538 \\
\hline $\mathrm{H} 30$ & 0.275901 & 0.159544 \\
\hline
\end{tabular}

\begin{tabular}{lll}
\hline RHF & & B3LYP \\
3-21G & & $\mathbf{6 - 3 1 G +}$ \\
Atoms & Charge $($ statC) & Charge $($ statC) \\
\hline C1 & -0.097663 & -0.097181 \\
C2 & -0.097893 & -0.098746 \\
C3 & -0.146332 & -0.047966 \\
C4 & -0.146135 & -0.047932 \\
C5 & -0.097993 & 0.019146 \\
C6 & -0.097829 & 0.019567 \\
C7 & -0.146167 & -0.047671 \\
C8 & -0.146164 & -0.048182 \\
H9 & 0.240060 & 0.183377 \\
H10 & 0.240064 & 0.183389 \\
C11 & -0.097765 & 0.020386 \\
C12 & -0.097831 & 0.019587 \\
C13 & -0.189051 & -0.226265 \\
C14 & -0.189209 & -0.226150 \\
H15 & 0.240063 & 0.183385 \\
H16 & 0.240066 & 0.183386 \\
C17 & -0.189195 & -0.226984 \\
C18 & -0.189211 & -0.226340 \\
C19 & -0.240755 & -0.236637 \\
C20 & -0.240731 & -0.236236 \\
H21 & 0.240668 & 0.181051 \\
H22 & 0.240668 & 0.181045 \\
C23 & -0.240753 & -0.236079 \\
C24 & -0.240732 & -0.236280 \\
H25 & 0.240669 & 0.181044 \\
H26 & 0.240666 & 0.181044 \\
H27 & 0.242115 & 0.175555 \\
H28 & 0.242124 & 0.175563 \\
H29 & 0.242124 & 0.175561 \\
H30 & 0.242122 & 0.175563 \\
\hline & & \\
\hline
\end{tabular}

Vibrational Frequencies and IR Intensities in Gas Phase and in Methanol

Spectroscopy is the study of matter and its interaction with electromagnetic radiation. All matter contains molecules; these molecules have bonds that are continually vibrating and moving around. These bonds can vibrate with stretched motions or bent motions (Mukamel, 2000). Infrared spectroscopy is the study of how molecules absorb infrared radiation and ultimately convert it to heat. Thus, IR spectra is the study of interaction of infrared light with matter. When a molecule absorbs infrared radiation, its chemical bonds vibrate and may either be stretched, contracted or bent (Villar et al, 2012). The main focus of vibrational analysis is to get vibrational modes connected with the precise molecular structures of the measured compound (Hamm et. al., 1998). Tables 8 and 9 show the calculated vibrational frequencies and intensities of the Tetracene molecule in the gas phase and in methanol.

From the values of the vibrational frequencies obtained in methanol, the first frequencies for DFT were negative which shows that the molecule was at first order saddle point or transition state on the potential energy surface. Whereas at RHF, where no imaginary frequencies exist, the molecule was very stable. However, in the gas phase, no imaginary frequencies were found at both levels of theory indicating that the molecule would be more stable than in methanol.

In gas phase, the most intense frequency was found to be about $925 \mathrm{~cm}^{-1}$ which occurred at an intensity of $146.7973 \mathrm{~km} / \mathrm{mol}$. At this frequency there is strong P$\mathrm{OR}$ esters and medium $\mathrm{RCOOH} \mathrm{O}-\mathrm{H}$ bend mode of vibrations observed. The second most intense peak has frequency of about $1115 \mathrm{~cm}^{-1}$ which occurred at the intensity of $113.4457 \mathrm{~km} / \mathrm{mol}$. At this frequency, strong C-F stretch, strong C-O stretch, medium C-N stretch and weak $\mathrm{P}=\mathrm{O}$ phosphate mode of vibrations were observed. The third most intense frequency of about $3228 \mathrm{~cm}^{-1}$ was observed at an intensity of $72.3565 \mathrm{KM} / \mathrm{mole}$. At this frequency, strong (broad) dimer $\mathrm{OH}$, and strong broad ArO- $\mathrm{H} \mathrm{H}$-bonded mode of vibrations were observed. The fourth most intense peak has frequency of about $3226 \mathrm{~cm}^{-1}$ which occurred at the intensity of $47.321 \mathrm{~km} / \mathrm{mol}$. At this frequency, strong (broad) dimer $\mathrm{OH}$ and strong broad ArO-H Hbonded mode of vibrations were observed. IRPal 2.0 was used to interpret these frequencies. 
BAJOPAS Volume 10 Number 1 June, 2017

In Methanol, the most intense frequency was found to be about $390.1077 \mathrm{~cm}^{-1}$ which occurred at the intensity of $474.126 \mathrm{~km} / \mathrm{mol}$ At this frequency, strong $\mathrm{C}-\mathrm{Br}$ and weak $\mathrm{S}-\mathrm{S}$ disulfide asymmetric mode of vibrations were observed. The second most intense frequency of about $863 \mathrm{~cm}^{-1}$ was observed at an intensity of $201.4069 \mathrm{~km} / \mathrm{mol}$. At this frequency, there are medium $\mathrm{C}-\mathrm{H}$ out of plane, strong S-OR esters and strong (broad) $\mathrm{N}-\mathrm{H}$ wag amines mode of vibrations observed. Another most intense peak has frequency of about $865 \mathrm{~cm}^{-1}$ which occurred at the intensity of $198.1175 \mathrm{~km} / \mathrm{mol}$. At this frequency, medium C-H out of plane and strong (broad) $\mathrm{N}-\mathrm{H}$ wag amines mode of vibrations were observed. Another most intense peak has frequency of about $1088.5374 \mathrm{~cm}^{-1}$ at the intensity of $187.2281 \mathrm{~km} / \mathrm{mol}$. At this frequency, there are strong stretch, strong C-O stretch, medium C-N stretch and weak $\mathrm{P}-\mathrm{H}$ bending mode of vibrations. IRPal 2.0 software was used to interpret these frequencies.

Table 8: Vibrational Frequencies and Intensities of Tetracene Molecule in Gas Phase

\begin{tabular}{|c|c|c|c|c|c|c|c|c|}
\hline \multirow{3}{*}{ Mode } & \multicolumn{4}{|c|}{ RHF } & \multicolumn{4}{|c|}{ B3LYP } \\
\hline & \multicolumn{2}{|l|}{$3-21 G$} & \multicolumn{2}{|c|}{$6-31 G$} & \multicolumn{2}{|c|}{$3-21 G$} & \multicolumn{2}{|c|}{$6-31 G$} \\
\hline & $\begin{array}{l}\text { Frequency } \\
\left(\mathrm{cm}^{-1}\right)\end{array}$ & $\begin{array}{l}\text { Intensity } \\
(\mathrm{km} / \mathrm{mol})\end{array}$ & $\begin{array}{l}\text { Frequency } \\
\left(\mathrm{cm}^{-1}\right)\end{array}$ & $\begin{array}{l}\text { Intensity } \\
(\mathrm{km} / \mathrm{mol})\end{array}$ & $\begin{array}{l}\text { Frequency } \\
\left(\mathrm{cm}^{-1}\right)\end{array}$ & $\begin{array}{l}\text { Intensity } \\
(\mathrm{km} / \mathrm{mol})\end{array}$ & $\begin{array}{l}\text { Frequency } \\
\left(\mathrm{cm}^{-1}\right)\end{array}$ & $\begin{array}{l}\text { Intensity } \\
(\mathrm{km} / \mathrm{mol})\end{array}$ \\
\hline 1 & 75.6695 & 0.0000 & 56.2805 & 0.0000 & 60.8430 & 0.0000 & 76.4232 & 0.0000 \\
\hline 2 & 92.6427 & 0.7087 & 85.8971 & 0.5090 & 55.9206 & 0.7459 & 47.4678 & 0.4992 \\
\hline 3 & 187.9230 & 3.0238 & 184.4829 & 3.1824 & 158.0150 & 0.0000 & 153.0607 & 0.0000 \\
\hline 4 & 207.1355 & 0.0000 & 199.5840 & 0.0000 & 161.1667 & 1.5555 & 157.5221 & 1.5577 \\
\hline 5 & 207.9812 & 0.0000 & 203.1569 & 0.0000 & 162.4580 & 0.0000 & 160.0276 & 0.0000 \\
\hline 6 & 341.2376 & 0.0000 & 343.1608 & 0.0000 & 299.6296 & 1.3706 & 300.6415 & 0.8515 \\
\hline 7 & 350.8384 & 0.0000 & 346.7571 & 0.0000 & 305.4639 & 0.0006 & 304.5650 & 0.0009 \\
\hline 8 & 358.3268 & 0.0027 & 352.9137 & 0.0006 & 315.2871 & 0.0000 & 311.0685 & 0.0000 \\
\hline 9 & 360.7222 & 1.2829 & 357.7453 & 0.9339 & 326.9431 & 0.0000 & 327.9912 & 0.0000 \\
\hline 10 & 464.4543 & 0.0000 & 461.3269 & 0.0000 & 401.7233 & 0.0000 & 399.5172 & 0.0000 \\
\hline 11 & 518.7775 & 0.0044 & 513.7608 & 0.0011 & 470.8781 & 0.1375 & 465.8261 & 0.1327 \\
\hline 12 & 561.9690 & 0.0002 & 556.8645 & 0.0000 & 485.6955 & 11.7628 & 489.5307 & 10.7455 \\
\hline 13 & 562.5964 & 7.3444 & 560.4716 & 7.5839 & 488.1081 & 14.4726 & 495.7325 & 0.0000 \\
\hline 14 & 572.7510 & 0.2114 & 571.9502 & 0.0043 & 490.2315 & 0.0091 & 497.1328 & 8.0731 \\
\hline 15 & 573.1497 & 25.6793 & 573.9909 & 18.4977 & 522.8642 & 0.0001 & 517.9468 & 0.0000 \\
\hline 16 & 607.7163 & 0.0002 & 605.2988 & 0.0002 & 523.9449 & 0.0000 & 527.2253 & 0.0001 \\
\hline 17 & 611.2569 & 6.2553 & 614.6846 & 7.1597 & 586.6963 & 7.9413 & 585.4165 & 8.2483 \\
\hline 18 & 666.1458 & 0.0000 & 659.3767 & 0.0000 & 592.5708 & 0.0000 & 585.7186 & 0.0021 \\
\hline 19 & 688.6483 & 10.6211 & 685.2601 & 10.4330 & 647.2127 & 2.1495 & 641.6978 & 2.0416 \\
\hline 20 & 718.3705 & 0.0000 & 713.5350 & 0.0000 & 672.3330 & 0.0000 & 666.9348 & 0.0000 \\
\hline 21 & 726.3693 & 0.8831 & 720.8255 & 0.9683 & 678.7602 & 1.0234 & 673.0396 & 0.9722 \\
\hline 22 & 819.5215 & 0.3140 & 830.7329 & 0.0000 & 730.8192 & 0.0000 & 733.4112 & 0.0000 \\
\hline 23 & 833.9201 & 0.0000 & 849.3542 & 1.9150 & 768.7862 & 0.0003 & 771.6327 & 0.0002 \\
\hline 24 & 851.0213 & 0.0002 & 862.0756 & 0.0000 & 776.9470 & 0.0001 & 774.1840 & 0.0000 \\
\hline 25 & 870.2120 & 0.0000 & 872.8729 & 0.0000 & 780.6062 & 105.9127 & 783.8994 & 92.8634 \\
\hline 26 & 885.0783 & 0.0000 & 879.4206 & 0.0000 & 787.4798 & 0.0002 & 785.1093 & 0.0088 \\
\hline 27 & 901.4810 & 0.0000 & 896.3359 & 0.0000 & 813.4689 & 2.0032 & 819.2909 & 0.0001 \\
\hline 28 & 909.5190 & 5.1830 & 912.4693 & 0.0013 & 819.0863 & 0.0001 & 822.9273 & 1.9479 \\
\hline 29 & 914.6558 & 0.0013 & 922.7361 & 143.7864 & 822.1176 & 0.0001 & 826.1029 & 0.0003 \\
\hline 30 & 924.9862 & 146.7973 & 973.8468 & 0.0000 & 871.0476 & 0.0000 & 877.1978 & 0.0000 \\
\hline 31 & 956.5412 & 0.0000 & 1000.7977 & 1.2014 & 880.8517 & 0.0000 & 887.2268 & 0.0000 \\
\hline 32 & 1006.8840 & 0.0001 & 1008.4999 & 0.0022 & 885.0250 & 0.0000 & 888.7298 & 0.0000 \\
\hline 33 & 1024.3309 & 0.0000 & 1023.2253 & 0.0000 & 915.4883 & 0.0000 & 926.2123 & 0.0000 \\
\hline 34 & 1033.7174 & 0.0000 & 1032.7025 & 0.0000 & 917.6460 & 0.0000 & 929.7441 & 0.0000 \\
\hline 35 & 1050.7178 & 0.0000 & 1048.9363 & 0.0000 & 941.5385 & 89.3633 & 937.2543 & 0.0000 \\
\hline 36 & 1053.6352 & 9.6136 & 1050.9970 & 9.4360 & 943.0296 & 0.0005 & 946.3282 & 77.1539 \\
\hline 37 & 1081.3464 & 0.0000 & 1084.0315 & 0.0000 & 945.2638 & 0.0012 & 960.2049 & 0.0001 \\
\hline 38 & 1114.4980 & 113.4457 & 1113.8769 & 115.1405 & 985.1732 & 2.3774 & 976.0189 & 2.3482 \\
\hline 39 & 1121.7868 & 0.0002 & 1128.7643 & 0.0001 & 996.6672 & 0.0000 & 991.2499 & 0.7938 \\
\hline 40 & 1122.8851 & 4.4718 & 1144.6443 & 5.2867 & 998.8674 & 14.9108 & 995.1016 & 22.2836 \\
\hline 41 & 1125.0030 & 0.0005 & 1146.4864 & 0.0006 & 1031.5744 & 0.0001 & 1027.2202 & 0.0001 \\
\hline 42 & 1171.2719 & 0.0001 & 1167.0585 & 0.0000 & 1031.7471 & 0.0001 & 1027.8227 & 0.0001 \\
\hline 43 & 1174.3920 & 24.5417 & 1171.0136 & 31.6815 & 1076.5581 & 5.4585 & 1090.1132 & 6.3039 \\
\hline 44 & 1201.0421 & 6.2710 & 1203.7112 & 0.0001 & 1076.8946 & 0.0054 & 1090.3157 & 0.0179 \\
\hline 45 & 1203.6088 & 0.0002 & 1204.2953 & 0.0001 & 1149.7175 & 5.0099 & 1159.8028 & 5.3672 \\
\hline
\end{tabular}




\begin{tabular}{|c|c|c|c|c|c|c|c|c|}
\hline \multirow[b]{3}{*}{ Mode } & \multicolumn{4}{|c|}{ RHF } & \multicolumn{4}{|c|}{ B3LYP } \\
\hline & \multicolumn{2}{|c|}{$3-21 G$} & \multicolumn{2}{|c|}{$6-31 G$} & \multicolumn{2}{|c|}{$3-21 G$} & \multicolumn{2}{|c|}{$6-31 G$} \\
\hline & $\begin{array}{l}\text { Frequency } \\
\left(\mathrm{cm}^{-1}\right)\end{array}$ & $\begin{array}{l}\text { Intensity } \\
(\mathrm{km} / \mathrm{mol})\end{array}$ & $\begin{array}{l}\text { Frequency } \\
\left(\mathrm{cm}^{-1}\right)\end{array}$ & $\begin{array}{l}\text { Intensity } \\
(\mathrm{km} / \mathrm{mol})\end{array}$ & $\begin{array}{l}\text { Frequency } \\
\left(\mathrm{cm}^{-1}\right)\end{array}$ & $\begin{array}{l}\text { Intensity } \\
(\mathrm{km} / \mathrm{mol})\end{array}$ & $\begin{array}{l}\text { Frequency } \\
\left(\mathrm{cm}^{-1}\right)\end{array}$ & $\begin{array}{l}\text { Intensity } \\
(\mathrm{km} / \mathrm{mol})\end{array}$ \\
\hline 47 & 1228.0843 & 0.0000 & 1241.2977 & 0.0000 & 1174.2296 & 0.0509 & 1206.1377 & 0.1951 \\
\hline 48 & 1291.5950 & 0.0001 & 1316.1287 & 0.0000 & 1240.1708 & 0.0000 & 1231.8164 & 0.0000 \\
\hline 49 & 1333.1109 & 0.5643 & 1335.1641 & 0.4239 & 1245.0785 & 0.9611 & 1240.1336 & 1.1601 \\
\hline 50 & 1344.8399 & 0.0120 & 1355.7454 & 0.0001 & 1256.9308 & 0.0001 & 1257.3737 & 0.0001 \\
\hline 51 & 1345.6901 & 0.0175 & 1363.9028 & 0.1933 & 1271.7312 & 0.0001 & 1279.2583 & 0.0817 \\
\hline 52 & 1346.0218 & 0.5299 & 1365.9530 & 0.0001 & 1280.9731 & 0.0001 & 1290.7578 & 0.0001 \\
\hline 53 & 1393.7309 & 0.0140 & 1423.8088 & 0.0064 & 1334.2248 & 0.0026 & 1340.6704 & 0.0018 \\
\hline 54 & 1408.5003 & 0.0028 & 1424.3495 & 0.0103 & 1336.9371 & 0.0370 & 1346.3264 & 0.0225 \\
\hline 55 & 1425.1873 & 7.9944 & 1439.6417 & 5.1020 & 1345.7677 & 1.9744 & 1357.7771 & 0.1288 \\
\hline 56 & 1446.5942 & 9.6160 & 1449.8056 & 14.1745 & 1353.5709 & 18.5786 & 1358.4688 & 20.7360 \\
\hline 57 & 1448.5680 & 14.8035 & 1451.4662 & 15.0375 & 1371.7520 & 15.1400 & 1378.3768 & 0.0032 \\
\hline 58 & 1464.8907 & 0.0008 & 1480.9103 & 0.0003 & 1373.6588 & 0.1573 & 1399.5513 & 11.8050 \\
\hline 59 & 1471.5965 & 0.0003 & 1510.7427 & 0.0002 & 1421.8969 & 0.0001 & 1477.0983 & 5.9877 \\
\hline 60 & 1494.1931 & 1.8857 & 1568.4638 & 2.3926 & 1466.3713 & 2.5794 & 1478.3358 & 0.0005 \\
\hline 61 & 1507.6691 & 0.0007 & 1578.2416 & 0.0007 & 1473.2364 & 0.0008 & 1511.7511 & 0.0001 \\
\hline 62 & 1588.4187 & 3.6684 & 1592.4816 & 4.4876 & 1476.2162 & 5.5372 & 1519.6777 & 0.0013 \\
\hline 63 & 1616.2771 & 0.0000 & 1627.1036 & 0.0001 & 1511.9136 & 0.0001 & 1525.1774 & 2.2136 \\
\hline 64 & 1647.7492 & 0.0000 & 1654.4165 & 0.0000 & 1539.9075 & 0.0000 & 1563.4515 & 0.0003 \\
\hline 65 & 1662.0585 & 18.7208 & 1683.7949 & 1.9024 & 1562.2533 & 3.9007 & 1580.3779 & 3.2581 \\
\hline 66 & 1665.7993 & 1.8934 & 1687.2065 & 14.7786 & 1566.0620 & 10.5868 & 1591.1559 & 6.8071 \\
\hline 67 & 1694.3167 & 0.0018 & 1730.9724 & 0.0009 & 1601.0169 & 0.0008 & 1631.9770 & 0.0004 \\
\hline 68 & 1718.0578 & 0.0018 & 1760.6674 & 0.0014 & 1622.6562 & 0.0019 & 1659.5786 & 0.0009 \\
\hline 69 & 1723.8560 & 6.4557 & 1768.1054 & 6.5970 & 1626.2588 & 2.0521 & 1664.4215 & 1.6165 \\
\hline 70 & 1726.6887 & 0.0015 & 1776.4761 & 0.0010 & 1633.3695 & 0.0010 & 1676.7261 & 0.0009 \\
\hline 71 & 1750.7824 & 0.0083 & 1805.1828 & 0.0361 & 1634.2921 & 0.0007 & 1680.4959 & 0.0004 \\
\hline 72 & 1756.9810 & 25.7739 & 1808.0353 & 22.2400 & 1653.3774 & 7.8908 & 1696.3615 & 6.3086 \\
\hline 73 & 3183.3616 & 0.0000 & 3202.7526 & 0.0000 & 3137.6595 & 0.0000 & 3156.6657 & 0.0000 \\
\hline 74 & 3185.4751 & 1.3874 & 3204.8192 & 1.7820 & 3139.7705 & 1.2290 & 3158.7936 & 1.6990 \\
\hline 75 & 3189.1264 & 9.2173 & 3208.2650 & 17.8349 & 3141.6380 & 16.2330 & 3160.3750 & 31.1390 \\
\hline 76 & 3191.8321 & 0.0001 & 3211.0957 & 0.0001 & 3144.1933 & 0.0000 & 3163.2153 & 0.0001 \\
\hline 77 & 3202.6558 & 5.7130 & 3219.9535 & 10.9950 & 3156.4084 & 0.0061 & 3173.6550 & 0.0272 \\
\hline 78 & 3202.8069 & 0.0007 & 3220.1097 & 0.0039 & 3156.4509 & 14.2584 & 3173.7075 & 24.5772 \\
\hline 79 & 3205.7724 & 5.4234 & 3223.7841 & 4.8693 & 3158.7980 & 5.4436 & 3176.6969 & 5.4351 \\
\hline 80 & 3206.4747 & 0.0001 & 3224.6236 & 0.0002 & 3159.2023 & 0.0000 & 3177.2038 & 0.0001 \\
\hline 81 & 3227.5948 & 3.0247 & 3244.8604 & 0.0050 & 3181.2489 & 0.1871 & 3198.5211 & 0.0131 \\
\hline 82 & 3227.6215 & 47.2311 & 3244.9226 & 73.0343 & 3181.2534 & 35.6343 & 3198.5499 & 62.1495 \\
\hline 83 & 3248.3453 & 72.3565 & 3266.9563 & 116.1227 & 3199.7416 & 63.1105 & 3218.5891 & 108.2845 \\
\hline 84 & 3248.6157 & 0.0018 & 3267.3411 & 0.0014 & 3199.9455 & 0.0006 & 3218.9013 & 0.0003 \\
\hline
\end{tabular}

Table 9: Vibratonal Frequencies and Intensities of Tetracene Molecule in Methanol.

\begin{tabular}{lllllllll} 
& & & \multicolumn{3}{c}{ RHF } & \multicolumn{2}{c}{$\mathbf{6 - 3 1 G}$} & \multicolumn{3}{c}{ 3-21G } & \multicolumn{2}{c}{ B3LYP } \\
Mode & $\begin{array}{r}\text { Frequency } \\
\left(\mathrm{cm}^{-1}\right)\end{array}$ & $\begin{array}{l}\text { Intensity } \\
(\mathrm{km} / \mathrm{mol})\end{array}$ & $\begin{array}{l}\text { Frequency } \\
\left(\mathrm{cm}^{-1}\right)\end{array}$ & $\begin{array}{l}\text { Intensity } \\
(\mathrm{km} / \mathrm{mol})\end{array}$ & $\begin{array}{l}\text { Frequency } \\
\left.\mathrm{cm}^{-1}\right)\end{array}$ & $\begin{array}{l}\text { Intensity } \\
(\mathrm{km} / \mathrm{mol})\end{array}$ & $\begin{array}{l}\text { Frequency } \\
\left(\mathrm{cm}^{-1}\right)\end{array}$ & $\begin{array}{l}\text { Intensity } \\
(\mathrm{km} / \mathrm{mol})\end{array}$ \\
\hline 1 & 65.9403 & 1.6433 & 63.5472 & 1.2546 & -61.7171 & 1.5152 & -60.0284 & 1.0042 \\
2 & 104.3894 & 0.0002 & 101.5053 & 0.0002 & 96.1945 & 0.0001 & 93.8897 & 0.0001 \\
3 & 170.0060 & 0.0001 & 169.0852 & 0.0001 & 155.1964 & 0.0002 & 156.4318 & 0.0002 \\
4 & 175.6798 & 2.8932 & 176.5595 & 3.4279 & 164.7723 & 0.0800 & 165.4692 & 2.3408 \\
5 & 214.8220 & 0.0001 & 213.1757 & 0.0000 & 198.6345 & 0.0001 & 198.9881 & 0.0000 \\
6 & 298.4672 & 1.6716 & 299.5439 & 1.1931 & 275.9632 & 1.7751 & 280.2008 & 1.0598 \\
7 & 335.2587 & 0.0000 & 334.2823 & 0.0000 & 311.0720 & 0.0000 & 310.7234 & 0.0000 \\
8 & 340.4258 & 0.0000 & 339.9878 & 0.0000 & 320.6282 & 0.0000 & 320.0591 & 0.0000 \\
9 & 356.7476 & 0.0001 & 354.8946 & 0.0000 & 326.8650 & 0.0000 & 328.0300 & 0.0000 \\
10 & 426.0629 & 0.0000 & 425.4724 & 0.0001 & 0.1170 & 458.8012 & 390.7004 & 0.0000 \\
11 & 496.2697 & 0.0101 & 492.4624 & 0.0012 & 390.1077 & 474.1260 & 456.3864 & 0.1328 \\
12 & 526.8400 & 85.9214 & 529.5507 & 72.5912 & 474.1260 & 53.8238 & 484.3646 & 42.3109 \\
13 & 534.4352 & 0.0001 & 536.3439 & 0.0001 & 481.0749 & 0.0000 & 489.3288 & 0.0000 \\
14 & 550.5677 & 0.1011 & 550.8965 & 0.0019 & 487.9860 & 4.1601 & 495.3102 & 0.9519 \\
15 & 557.0304 & 0.0000 & 554.1061 & 0.0000 & 518.0187 & 0.0000 & 515.2024 & 0.0000 \\
16 & 577.2554 & 0.0004 & 578.2629 & 0.0003 & 525.8151 & 0.0003 & 531.6542 & 0.0002 \\
\hline
\end{tabular}


BAJOPAS Volume 10 Number 1 June, 2017 Table 9 continue

\begin{tabular}{|c|c|c|c|c|c|c|c|c|}
\hline \multirow[b]{3}{*}{ Mode } & \multicolumn{4}{|c|}{ RHF } & \multicolumn{4}{|c|}{ B3LYP } \\
\hline & \multicolumn{2}{|c|}{ 3-21G } & \multicolumn{2}{|c|}{$6-31 G$} & \multicolumn{2}{|c|}{$3-21 G$} & \multicolumn{2}{|c|}{ 6-31G } \\
\hline & $\begin{array}{l}\text { Frequency } \\
\left(\mathrm{cm}^{-1}\right)\end{array}$ & $\begin{array}{l}\text { Intensity } \\
(\mathrm{km} / \mathrm{mol})\end{array}$ & $\begin{array}{l}\text { Frequency } \\
\left(\mathrm{cm}^{-1}\right)\end{array}$ & $\begin{array}{l}\text { Intensity } \\
(\mathrm{km} / \mathrm{mol})\end{array}$ & $\begin{array}{l}\text { Frequency } \\
\left.\mathrm{cm}^{-1}\right)\end{array}$ & $\begin{array}{l}\text { Intensity } \\
(\mathrm{km} / \mathrm{mol})\end{array}$ & $\begin{array}{l}\text { Frequency } \\
\left(\mathrm{cm}^{-1}\right)\end{array}$ & $\begin{array}{l}\text { Intensity } \\
(\mathrm{km} / \mathrm{mol})\end{array}$ \\
\hline 17 & 611.3406 & 10.4052 & 608.2750 & 10.9314 & 573.1936 & 15.1059 & 570.4339 & 15.5773 \\
\hline 18 & 634.9135 & 0.0000 & 630.1016 & 0.0000 & 584.6554 & 0.0000 & 580.5804 & 0.0000 \\
\hline 19 & 668.9787 & 11.9346 & 669.1502 & 11.9324 & 630.4100 & 3.6157 & 628.5219 & 3.4056 \\
\hline 20 & 692.3884 & 0.0000 & 689.8150 & 0.0000 & 647.0709 & 0.0000 & 644.4719 & 0.0000 \\
\hline 21 & 707.1946 & 2.0796 & 703.3919 & 2.0659 & 657.9946 & 1.9656 & 654.8323 & 2.0477 \\
\hline 22 & 762.7755 & 1.4279 & 787.8042 & 3.1533 & 742.9551 & 0.0000 & 746.8473 & 0.0000 \\
\hline 23 & 812.8106 & 0.0000 & 823.8376 & 0.0000 & 745.6495 & 2.5506 & 759.2788 & 3.1413 \\
\hline 24 & 835.7624 & 0.0001 & 832.1762 & 0.0001 & 754.6934 & 0.0000 & 764.7443 & 0.0000 \\
\hline 25 & 852.2046 & 0.0000 & 850.1988 & 0.0000 & 758.7332 & 0.0000 & 765.5827 & 0.0000 \\
\hline 26 & 853.2378 & 0.0000 & 857.3735 & 0.0000 & 771.0841 & 151.6191 & 778.2413 & 139.3628 \\
\hline 27 & 864.8877 & 198.1175 & 862.6598 & 201.4069 & 783.5573 & 0.0004 & 781.9944 & 0.0002 \\
\hline 28 & 875.9888 & 0.0000 & 869.4559 & 0.0001 & 792.5597 & 0.0000 & 790.9094 & 0.0000 \\
\hline 29 & 890.1298 & 0.0000 & 882.5929 & 0.0000 & 799.2529 & 0.0000 & 799.5257 & 0.0000 \\
\hline 30 & 912.9082 & 0.0000 & 928.5097 & 0.0000 & 858.1184 & 0.0000 & 870.7280 & 0.0000 \\
\hline 31 & 975.7099 & 0.0000 & 972.7980 & 0.0000 & 867.2656 & 0.0000 & 874.0809 & 0.0000 \\
\hline 32 & 983.0928 & 0.0001 & 980.6177 & 0.0000 & 874.6317 & 0.0000 & 882.3976 & 0.0000 \\
\hline 33 & 1001.8938 & 0.0000 & 1001.4704 & 0.0000 & 888.3667 & 0.0000 & 892.2106 & 0.0000 \\
\hline 34 & 1029.7773 & 0.0000 & 1024.7324 & 0.0000 & 915.1815 & 0.0003 & 922.9333 & 0.0000 \\
\hline 35 & 1031.0676 & 7.6401 & 1040.3844 & 14.2760 & 927.6901 & 0.0000 & 924.8769 & 0.0001 \\
\hline 36 & 1044.0444 & 13.9690 & 1055.4251 & 0.0000 & 941.3440 & 143.9040 & 946.6025 & 138.2008 \\
\hline 37 & 1048.6446 & 0.0000 & 1067.2016 & 5.3807 & 943.7424 & 0.0000 & 955.7919 & 0.0000 \\
\hline 38 & 1056.9567 & 0.0001 & 1079.8287 & 0.0000 & 967.3464 & 5.0057 & 959.7271 & 5.0690 \\
\hline 39 & 1092.8134 & 170.1864 & 1088.5374 & 187.2281 & 1003.4686 & 0.0000 & 998.7773 & 0.0000 \\
\hline 40 & 1102.8570 & 0.0000 & 1102.9875 & 0.0000 & 1004.4480 & 14.5676 & 1000.6011 & 22.2337 \\
\hline 41 & 1119.4012 & 0.7145 & 1148.9339 & 0.0001 & 1011.5808 & 5.1424 & 1029.8416 & 0.0005 \\
\hline 42 & 1156.7998 & 0.0001 & 1150.8871 & 29.8210 & 1012.4524 & 0.0003 & 1030.2022 & 0.0232 \\
\hline 43 & 1158.3420 & 22.1757 & 1157.4505 & 0.4788 & 1032.3471 & 0.0000 & 1030.9573 & 5.6923 \\
\hline 44 & 1182.9375 & 0.0000 & 1179.9321 & 0.0000 & 1032.4531 & 0.0000 & 1031.4885 & 0.0002 \\
\hline 45 & 1183.1881 & 0.0002 & 1180.3246 & 0.0001 & 1152.2236 & 5.5843 & 1161.4175 & 5.9264 \\
\hline 46 & 1217.6095 & 9.9409 & 1238.8780 & 11.5871 & 1161.0725 & 0.0000 & 1164.6434 & 0.0000 \\
\hline 47 & 1241.3647 & 0.0000 & 1251.0120 & 0.0000 & 1167.7971 & 1.2581 & 1190.1632 & 4.1511 \\
\hline 48 & 1286.8325 & 0.0000 & 1296.2914 & 0.0000 & 1207.4722 & 0.0000 & 1207.0867 & 0.0000 \\
\hline 49 & 1298.3017 & 2.7466 & 1299.0911 & 1.3460 & 1211.0938 & 1.7328 & 1208.8055 & 2.9187 \\
\hline 50 & 1325.1267 & 0.0000 & 1327.9538 & 0.0000 & 1238.6841 & 0.0000 & 1237.3958 & 0.0000 \\
\hline 51 & 1329.5214 & 1.1285 & 1332.1292 & 0.8050 & 1241.5228 & 0.0000 & 1247.8049 & 0.2350 \\
\hline 52 & 1329.9905 & 0.0002 & 1334.2722 & 0.0000 & 1243.3848 & 0.2724 & 1252.2318 & 0.0000 \\
\hline 53 & 1382.5388 & 12.7565 & 1410.0319 & 18.2905 & 1321.6256 & 41.4754 & 1322.1373 & 0.0000 \\
\hline 54 & 1413.5084 & 29.2231 & 1423.7840 & 0.0001 & 1321.9787 & 2.6366 & 1327.7179 & 27.8111 \\
\hline 55 & 1426.1490 & 0.0000 & 1425.4423 & 25.8298 & 1323.7697 & 0.0000 & 1335.3770 & 17.3012 \\
\hline 56 & 1441.1866 & 22.3628 & 1443.2697 & 5.6010 & 1338.1971 & 3.7734 & 1340.8154 & 1.5840 \\
\hline 57 & 1449.4963 & 2.4017 & 1448.9771 & 16.2923 & 1364.4044 & 38.2259 & 1381.6352 & 0.0000 \\
\hline 58 & 1462.9684 & 0.0001 & 1493.2152 & 0.0000 & 1378.4352 & 0.0000 & 1391.5480 & 30.2454 \\
\hline 59 & 1486.3476 & 0.0000 & 1519.2777 & 0.0000 & 1383.2384 & 0.0000 & 1431.1775 & 0.0000 \\
\hline 60 & 1545.3725 & 0.0000 & 1554.1345 & 4.8458 & 1416.3897 & 3.4192 & 1445.4247 & 9.7113 \\
\hline 61 & 1549.5463 & 7.7489 & 1576.1985 & 0.0000 & 1417.2527 & 0.0000 & 1449.6704 & 0.0000 \\
\hline 62 & 1552.8718 & 4.5246 & 1585.3940 & 2.6095 & 1446.9039 & 9.8386 & 1451.5326 & 0.0005 \\
\hline 63 & 1594.9916 & 0.0000 & 1612.6128 & 0.0000 & 1490.2801 & 0.0000 & 1500.2062 & 0.0000 \\
\hline 64 & 1604.3431 & 0.0001 & 1613.2604 & 0.0000 & 1494.9627 & 0.0000 & 1502.2383 & 0.0000 \\
\hline 65 & 1612.0338 & 17.0229 & 1627.8805 & 12.4841 & 1508.9277 & 21.4157 & 1521.6332 & 14.4307 \\
\hline 66 & 1686.6079 & 0.0000 & 1708.3085 & 0.0000 & 1549.8496 & 0.0000 & 1573.4253 & 0.0000 \\
\hline 67 & 1707.5241 & 0.0000 & 1738.0254 & 16.7709 & 1555.7108 & 0.0000 & 1586.9237 & 0.0000 \\
\hline 68 & 1710.4738 & 9.6904 & 1740.9946 & 0.0001 & 1568.4970 & 4.5015 & 1596.5246 & 7.6228 \\
\hline 69 & 1744.5357 & 0.8707 & 1764.6042 & 2.1923 & 1601.6478 & 3.7771 & 1620.9592 & 4.2633 \\
\hline 70 & 1783.0433 & 0.0000 & 1814.8307 & 0.0000 & 1629.7112 & 0.0000 & 1664.2879 & 0.0000 \\
\hline 71 & 1816.7212 & 0.0002 & 1845.2921 & 0.0001 & 1651.6386 & 0.0000 & 1674.7071 & 0.0000 \\
\hline 72 & 1824.6086 & 36.3172 & 1852.0569 & 30.7307 & 1661.6186 & 23.2315 & 1689.4019 & 16.3068 \\
\hline 73 & 3291.9954 & 0.0000 & 3302.6135 & 0.0000 & 3128.4323 & 0.0000 & 3140.2708 & 0.0000 \\
\hline 74 & 3293.9965 & 1.1666 & 3304.6177 & 1.6286 & 3130.4255 & 0.7172 & 3142.3143 & 1.3007 \\
\hline 75 & 3296.1813 & 0.7812 & 3306.0896 & 2.4302 & 3131.6972 & 0.1442 & 3143.0801 & 5.1239 \\
\hline
\end{tabular}




\begin{tabular}{|c|c|c|c|c|c|c|c|c|}
\hline \multirow{3}{*}{ Mode } & \multicolumn{4}{|c|}{ RHF } & \multicolumn{4}{|c|}{ B3LYP } \\
\hline & \multicolumn{2}{|c|}{$3-21 G$} & \multicolumn{2}{|c|}{$6-31 G$} & \multicolumn{2}{|c|}{$3-21 G$} & \multicolumn{2}{|c|}{$6-31 G$} \\
\hline & $\begin{array}{l}\text { Frequency } \\
\left(\mathrm{cm}^{-1}\right)\end{array}$ & $\begin{array}{l}\text { Intensity } \\
(\mathrm{km} / \mathrm{mol})\end{array}$ & $\begin{array}{l}\text { Frequency } \\
\left(\mathrm{cm}^{-1}\right)\end{array}$ & $\begin{array}{l}\text { Intensity } \\
(\mathrm{km} / \mathrm{mol})\end{array}$ & $\begin{array}{l}\text { Frequency } \\
\left.\mathrm{cm}^{-1}\right)\end{array}$ & $\begin{array}{l}\text { Intensity } \\
(\mathrm{km} / \mathrm{mol})\end{array}$ & $\begin{array}{l}\text { Frequency } \\
\left(\mathrm{cm}^{-1}\right)\end{array}$ & $\begin{array}{l}\text { Intensity } \\
(\mathrm{km} / \mathrm{mol})\end{array}$ \\
\hline 76 & 3298.2231 & 0.0000 & 3308.3923 & 0.0000 & 3133.5125 & 0.0000 & 3145.1849 & 0.0000 \\
\hline 77 & 3301.6944 & 8.7199 & 3308.7474 & 0.0000 & 3138.9097 & 0.0128 & 3146.6547 & 0.0001 \\
\hline 78 & 3301.7518 & 0.0059 & 3309.0538 & 0.6935 & 3138.9194 & 1.1773 & 3146.7678 & 1.5903 \\
\hline 79 & 3304.7759 & 10.4108 & 3312.7454 & 7.4096 & 3141.2192 & 8.0121 & 3149.9650 & 6.8743 \\
\hline 80 & 3305.3161 & 0.0001 & 3313.5922 & 0.0000 & 3141.4629 & 0.0002 & 3150.3945 & 0.0001 \\
\hline 81 & 3321.3662 & 3.7444 & 3329.1188 & 0.0314 & 3156.8706 & 8.5082 & 3165.0512 & 32.7862 \\
\hline 82 & 3321.3677 & 9.5537 & 3329.1386 & 36.6543 & 3156.8916 & 0.0011 & 3165.0534 & 0.3408 \\
\hline 83 & 3333.5513 & 12.0362 & 3341.8215 & 42.6573 & 3168.6687 & 5.5319 & 3177.7177 & 34.8536 \\
\hline 84 & 3333.6158 & 0.0009 & 3341.9557 & 0.0011 & 3168.6990 & 0.0005 & 3177.8109 & 0.0009 \\
\hline
\end{tabular}

\section{CONCLUSION}

The optimized parameters (bond lengths and bond angles) of the tetracene molecule were calculated. The results obtained showed that the lowest average value of bond length was $1.072 \AA$ at RHF/3-21G and at DFT was $1.085 \AA$ in the gas phase. In methanol, the lowest average value was obtained to be $1.075 \AA$ using RHF and at DFT level, it was $1.087 \AA$. Optimized bond angle was found to be approximately $120^{\circ}$ in both gas phase and methanol suggesting that the molecule is planar benzene in which the $\mathrm{C}$ atoms are $s p^{2}$ hybridized. The calculated HOMO-LUMO Energy gap showed that the molecule will be more more stable in chemical reaction in the gas phase than in methanol. The DFT values of HOMO-LUMO gap appeared to be closer to the reported experimental value of $2.63 \mathrm{eV}$ than those obtained by RHF method. The mulliken atomic charges were found to be very sensitive to the basis sets used at both levels of theory. The results also indicated that at RHF/3-21G, all the hydrogen atoms exhibited positive charge values while all the carbon atoms exhibited negative charge values. The vibrational frequencies and intensities were calculated and the results obtained show that tetracene molecule would be more stable in the gas phase than in methanol as a result of the fact that no imaginary frequencies were observed in the gas phase. It is also found that, the most intense frequency was $924.9862 \mathrm{~cm}^{-1}$ which occurred at the intensity of $146.797 \mathrm{~km} / \mathrm{mol}$ with RHF/3-21G in gas

\section{REFERENCES}

Anna K. and Heinz B. (2015), "Electronic Processes in Organic Semiconductors" First Edition.WileyVCH Verlag GmbH \& Co. KGaA. Published byWiley-VCH Verlag GmbH \& Co. KGaA pp

Cioslowski, J. (1998) "Encyclopedia of Computational Chemistry", ed. P. v. R. Schleyer, N. L. Allinger, T. Clark, J. Gasteiger, P. A. Kollman, H. F. Schaefer III, Wiley, New York, vol. 5, p. 892

Denniston, K. J., Topping, J., and Dwyer, T. M, (2007) "General organic and Biochemistry", $5^{\text {th }}$ Edition, Towson University.

Frisch M. J., Trucks, G.W., Schlegel H. B., Scuseria G. E., Robb M. A., Cheeseman J. R., Montgomery J. A. Jr., Vreven, T. , Kudin K. N., Burant, J. C., Millam, J. M. , Iyengar S. S. phase while at $B 3 L Y P / 3-21 G$ has the most intense frequency of $474.1260 \mathrm{~cm}^{-1}$ occurring at an intensity of $390.107 \mathrm{~km} / \mathrm{mol}$ in methanol.

\section{RECOMMENDATIONS}

The authors recommend that other levels of theory and higher basis sets should be used to carry out the computations. Another molecular modelling tool can also be used to perform all the calculations and also to compute Raman frequencies and spectra of the molecule. Comparison should be made with this work.

\section{CONTRIBUTIONS OF AUTHORS}

A.S Gidado initiated the work, provided some relevant journals and performed the optimization calculations in both gas and methanol. Abubakar Maigari carried out the calculations for vibrational frequencies in both gas and methanol and also provided some relevant journals for review while G.S.M Galadanci did the calculations of HOMO-LUMO energy gaps and Mulliken atomic charges of the molecule in both gas and methanol. We all took part in the compilation and interpretation of the results as well as the proof reading of the entire write up.

\section{Acknowledgement}

The authors wish to thank Dr. Chifu E. Ndikilar of the department of Physics, Federal University Dutse, Jigawa state for providing them with the Guassian 03 package.

,Tomasi, J. , Barone, V. , Mennucci B., Cossi, M., Scalmani, G. , Rega, N. , Petersson, G. A., Nakatsuji , H., Hada, M., Ehara, M., Toyota, K., Fukuda, R., Hasegawa, J., Ishida, M., Nakajima, T., Honda, Y., Kitao, O., Nakai, H., Klene, M., Li, X., Knox, J. E., Hratchian, H. P., Cross, J. B., Bakken, V., Adamo, C., Jaramillo, J., Gomperts, R., Stratmann, R. E., Yazyev, O., Austin, A. J., Cammi, R., Pomelli, C., Ochterski, J. W.,Ayala, P. Y., Morokuma, K., Voth, G. A., Salvador, P., Dannenberg, J. J., Zakrzewski, V. G., Dapprich, S., Daniels, A.D., Strain, M. C., Farkas, O., Malick, D. K., Rabuck, A. D., Raghavachari, K., Foresman, J. B., Ortiz, J. V., Cui, Q., Baboul, A. G., Clifford, S., Cioslowski, J., Stefanov, B. B., Liu, G., Liashenko, A., Piskorz, P., Komaromi, 


\section{BAJOPAS Volume 10 Number 1 June, 2017}

I., Martin, R. L., Fox, D. J., Keith, T., AlLaham, M. A., Peng, C.Y., Nanayakkara, A., Challacombe, M., Gill, P.M.W., Johnson, B., Chen, W., Wong, M.W., Gonzalez, C., Pople, J. A. (2004)."Gaussian 03", Gaussian, Inc., Wallingford $\mathrm{C}$. T.

Gidado A.S, Bbabaji G. and Ado M. (2015), "Determination of Vibrational Frequencies, HOMO-LUMO Energy and IR-Spectra of Nucleobases (Adenine, Cytosine, Guanine, Thymine and Uracil)" J.NAMP vol 31 pp 465476.

Hamm, P. , Lim, M. and Hochstrasser, ,R .M (1998). "Structure of the amide 1 bond of peptides measured by Femtosecond nonlinear-infrared spectroscopy". J. Phys. Chem. B 102 (31): PP 6123.

Hoover, R. (2014). "Need to Track Organic NanoParticles Across the Universe? NASA's Got an App for That".NASA. Retrieved February 22, 2014.

Maksic, K. and Jug, Z. B. (1991). "Theoretical Model of Chemical Bonding", Ed. Z.B. Maksic, Part 3, Springer, Berlin , p. 29, p. 233.

Mukamel, S. (2000). "Multidimensional fentosecond correlation spectroscopies of Electronic and Vibrational Excitations". Annual Review of physical chemistry. 551(1) PP 691-729.

Mason, P. E. and Brady, J. W. (2007). Tetrahedrality and the Relationship between Collective Structure and Radial Distribution Functions in Liquid Water". J. Phys. Chem. Vol. 111 (20) pp 5669-5679.

Mason, Z-T. Zuh, J.T. R. Dieckmann, G.G. Malliara(2002) Appl. Phys, Lett, 81, pp. 4643-4645.

Musa, A. M. A., Saeed, A., Shaari, R. S. and Lawal, M. (2015). " Effects of delocalized $n$-electrons around the linear acenes ring ( $\mathrm{n}=1$ to 7 ): an electronic properties through DFT and quantum chemical descriptors", Molecular Physics: An International Journal at the Interface between Chemistry and Physics, DOI:10.1080/00268976.2014.993734.

Peter, I. D., Elizabeth I. M., Stephen, R. F. , Mark, E. T., (2009). "Measurement of the lowest unoccupied molecular orbital energies of molecular organic semiconductors" Organic Electronics 10 515-520.

Rubarani, P., Gangadharan and S. Sampath Krishnan 20013 "Natural Bond Orbital (NBO) Population Analysis of 1-Azanapthalene-8-01 ACTA Physical Vol. 125, PP 18-22.

Shaw and Seidler (2001) "Organic electronics: Introduction" IBM Res. \& Dev. Vol 45, PP 179-182

Suzuki S.; Morita Y.; Fukui K.; Sato K.; Shiomi D.; T.; Nakasuji K. (2006) 'Aromaticity on the pancake-bonded Dimer of Neutral phenalenyl Radical as studied by MS and NMR Spectroscopies and NICS Analysis' J. Am. Chem. Soc. 128 (8): 2530-2531.

Takahashi, T. T. Takenobu, J. Takeya, Y. I. (2007). "Ambipolar Light-Emitting Transistors of a Tetracene Single Crystal".Advanced Functional Materials 17 (10): 16231628. doi:10.1002/adfm.200700046.

Villar, A.; Gorritxategi, E. , Aranzade, E. ; Fernandex, L. A (2012). "Low-cost visiblenear infrared sensor for ol-line monitoring of fat and fatty acids content during the manufacturing process of the milk". Food Chemistry. 135(4): PP 2756-2760. Doi : $10.16 / \mathrm{j}$. food chem.. 2012.074. 